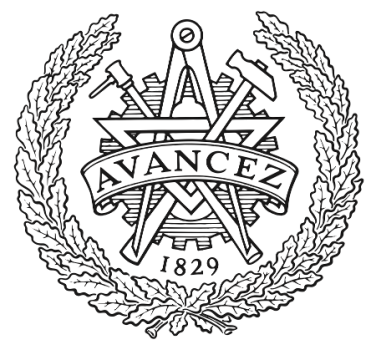

CHALMERS

UNIVERSITY OF TECHNOLOGY

\title{
Determination of Nanosized Adsorbate Mass in Solution Using Mechanical Resonators: Elimination of the so Far Inseparable Liquid Contribution
}

Downloaded from: https://research.chalmers.se, 2023-04-26 12:22 UTC

Citation for the original published paper (version of record):

Armanious, A., Agnarsson, B., Lundgren, A. et al (2021). Determination of Nanosized Adsorbate Mass in Solution Using Mechanical Resonators: Elimination of the so Far Inseparable Liquid Contribution. Journal of Physical Chemistry C, 125(41):

22733-22746. http://dx.doi.org/10.1021/acs.jpcc.1c04502

N.B. When citing this work, cite the original published paper. 


\title{
Determination of Nanosized Adsorbate Mass in Solution Using Mechanical Resonators: Elimination of the So Far Inseparable Liquid Contribution
}

\author{
Antonius Armanious,* Björn Agnarsson, Anders Lundgren, Vladimir P. Zhdanov, and Fredrik Höök*
}

Cite This: J. Phys. Chem. C 2021, 125, 22733-22746

Read Online

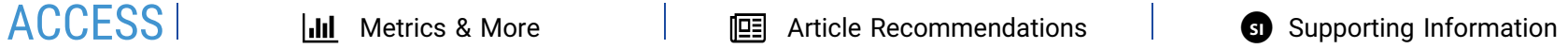

ABSTRACT: Assumption-free mass quantification of nanofilms, nanoparticles, and (supra)molecular adsorbates in a liquid environment remains a key challenge in many branches of science. Mechanical resonators can uniquely determine the mass of essentially any adsorbate; yet, when operating in a liquid environment, the liquid dynamically coupled to the adsorbate contributes significantly to the measured response, which complicates data interpretation and impairs quantitative adsorbate mass determination. Employing the Navier-Stokes equation for liquid velocity in contact with an oscillating surface, we show that the liquid contribution for rigid systems can be eliminated by measuring the response in solutions with identical kinematic

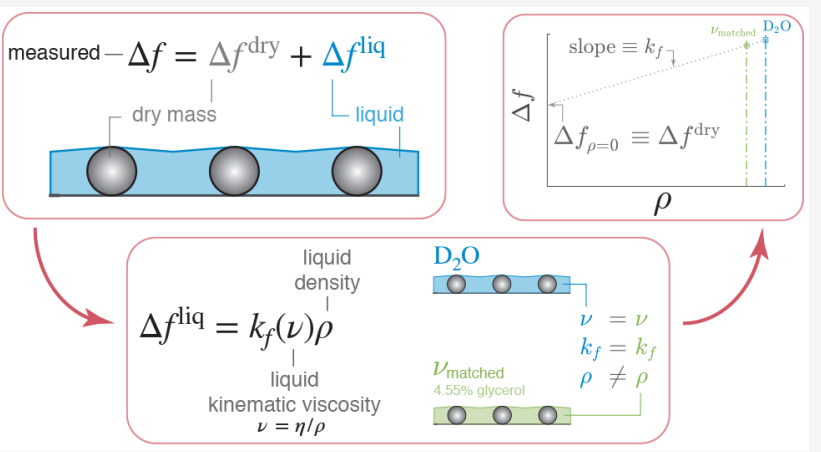
viscosity but different densities. Guided by this insight, we used the quartz crystal microbalance (QCM), one of the most widely employed mechanical resonators, to experimentally demonstrate that the kinematic-viscosity matching can be utilized to quantify the dry mass of rigid and in many cases also nonrigid adsorbate systems, including, e.g., rigid nanoparticles, tethered biological nanoparticles (lipid vesicles), as well as highly hydrated polymeric films. For all the adsorbates, the dry mass determined using the kinematic-viscosity matching was within the uncertainty limits of the corresponding mass determined using complementary methods, i.e., QCM in air, scanning electron microscopy, surface plasmon resonance, and theoretical estimations. The same approach applied to the simultaneously measured energy dissipation made it possible to quantify the mechanical properties of the adsorbate and its attachment to the surface, as demonstrated by, for example, probing the hydrodynamic stabilization induced by nanoparticle crowding. In addition to a unique means to quantify the liquid contribution to the measured response of mechanical resonators, we also envision that the kinematic-viscosity-matching approach will open up applications beyond mass determination, including a new means to investigate orientation, spatial distribution, and binding strength of adsorbates without the need for complementary techniques.

\section{INTRODUCTION}

Mass determination of nanosized adsorbates at solid-air and solid-liquid interfaces is central in many branches of science. Mechanical resonators are unique in their capacity to determine the mass of essentially any type of adsorbate at any surface without a priori knowledge of their physicochemical properties. In vacuum and in air, this is possible due to a direct proportionality between the measured changes in resonance frequency, $\Delta f$, and the adsorbed mass per unit area, $\Delta m$. For the quartz crystal microbalance (QCM), which is the most widely used mechanical resonator, this relationship is known as the Sauerbrey equation: ${ }^{1}$

$$
\Delta m=-C_{\mathrm{Q}} \cdot \Delta f_{n} / n
$$

where $n$ is the overtone number and $C_{Q}$ is the mass sensitivity constant, which for a $5 \mathrm{MHz}$ crystal is $18 \mathrm{ng} \cdot \mathrm{Hz}^{-1} \cdot \mathrm{cm}^{-2}$ (ref 2). QCM as well as many other mechanical resonators ${ }^{3-5}$ can also operate in a liquid environment and be further extended to simultaneously monitor both $\Delta f_{n}$ and changes in energy dissipation, $\Delta D_{n}{ }^{6,7}$ However, when operated in a liquid environment, not only the adsorbate but also the surrounding liquid becomes dynamically coupled to the oscillating crystal, a feature that is shared by all mechanical resonators operating in liquid. While this can in principle provide unique information about the physicochemical properties of an adsorbate, deconvoluting the entangled information in the measured response is an unresolved analytical challenge, as the measured response in a liquid environment is, in a complex manner, dependent on the mass of the adsorbate and the

Received: May 22, 2021

Revised: September 17, 2021

Published: October 8, 2021 
dynamically coupled liquid-a dependence that is manifested through changes in both $\Delta f_{n}$ and $\Delta D_{n}$ of the resonator. ${ }^{9}$

Still, the rich information contained in combined $\Delta f_{n}$ and $\Delta D_{n}$ measurements, especially when complemented with theoretical models representing the response for viscoelastic films, ${ }^{10-12}$ has turned out to be very valuable in multiple research areas, including hydration analysis of organic polymers, $^{13,14}$ proteins, ${ }^{15}$ and biological membranes. ${ }^{15,16}$ QCM has also been widely applied to monitor and characterize thin films, including porosity determination, ${ }^{17,18}$ monitoring the growth of mesoporous materials, ${ }^{19}$ probing responsiveness of polymeric coatings, ${ }^{20}$ and characterization of biomimetic membranes. ${ }^{21,22}$ Additionally, QCM has been extensively employed to study discrete adsorbates such as abiotic and biological macromolecules and nanoparticles (NPs). These studies included investigations of biomolecular interactions of proteins $^{23,24}$ and viruses; ${ }^{25,26}$ structure, $^{27,28}$ confirmation $^{29,30}$ and orientation changes ${ }^{31}$ of biomacromolecules; spatial distribution, ${ }^{32}$ size, ${ }^{33-35}$ deformation ${ }^{36,37}$ and dissolution ${ }^{38}$ of NPs; protein corona formation on amyloids; ${ }^{39}$ interactions between NPs and biomimetic membranes; ${ }^{40,41}$ as well as bioanalytical sensor development. ${ }^{42-46}$

Yet, the inseparable nature of the adsorbate and liquid contributions to the measured response puts a significant limitation on quantitative interpretation of adsorption measurements in a liquid environment. In particular, this shortcoming makes mass determination using mechanical resonators nonconclusive, unless complementary methods, such as surface plasmon resonance (SPR), ellipsometry, and/ or atomic force microscopy (AFM) are employed in parallel. $^{18,47-49}$

The use of combined experimental setups, e.g., QCM/SPR or QCM/AFM, comes with many challenges, such as operation at different spatial scales, requirement for very complex setups that are inaccessible for most users, and/or the need for pretreatment procedures that tend to change the properties of the samples under investigation. Moreover, mass determination using these complementary methods requires accurate knowledge, or determination, of the physical properties of adsorbates, such as refractive index or density. Accordingly, there has in recent years been an intense, albeit so far unsuccessful, search for a means to separate the contribution of coupled liquid using QCM measurements alone. ${ }^{2}$ Such a self-sufficient approach would obviate the need for complementary methods and provide quantitative information about the adsorbed dry mass and the coupled liquid contribution, as well as information about the dissipative energy loss associated with the adsorbate.

We here address this challenge through insights gained from the fundamental fluid dynamics principles of the NavierStokes equation for liquid velocity in contact with an oscillating surface $^{50}$ to show that the elimination of the liquid contribution to the sensed mass is possible by measuring the response in solutions with identical kinematic viscosity but different densities. To experimentally explore this insight, we used a QCM to investigate whether the kinematic-viscositymatching approach can be used to eliminate the liquid contribution and thus accurately determine the dry mass for a range of different nanosized adsorbates, including inorganic rigid nanoparticles (over a broad surface-coverage range), soft biological nanoparticles (lipid vesicles), and highly hydrated polymeric films. Comparisons with complementary dry-mass determination using the QCM operated in air as well as scanning electron microscopy (SEM), surface plasmon resonance (SPR), and theoretical predictions demonstrate excellent agreements, thus suggesting that the approach may offer a self-sufficient means to quantify the liquid contribution to the measured response of mechanical resonators enabling mass determination without the need for complementary techniques.

\section{METHODS}

Chemicals and Materials. Ethanol ( $\geq 99.5 \%)$ was purchased from Solveco (Roserberg, Sweden). Sodium dodecyl sulfate (SDS; $\geq 99.0 \%)$, anhydrous methanol (99.8\%), glycerol ( $\geq 99 \%), \mathrm{D}_{2} \mathrm{O}$ (99.9 atom \% D), DCl ( $\geq 99$ atom \% D), $\mathrm{NaOD}$ (40\%wt.), $\mathrm{HCl}(1 \mathrm{M}), \mathrm{NaCl}$ ( $\geq 99 \%), \mathrm{CaCl}_{2}$ anhydrous ( $\left.\geq 93 \%\right)$, phosphate buffered saline (PBS) tablets, bis(2-hydroxyethyl)amino-tris-(hydroxymethyl)-methane (Bis-Tris; $\geq 98 \%$ ), citric acid monohydrate $(\geq 98 \%)$, trisodium citrate dihydrate, poly-L-lysine (PLL; MW = 70 000-150 000), humic acids (HA), (3-aminopropyl)dimethylethoxysilane (97\%), $150 \mathrm{~nm} \mathrm{SiO}_{2}$ nanoparticles (NPs), anhydrous chloroform ( $\geq 99 \%)$, 1-palmitoyl-2-oleoylglycero-3-phosphocholine (POPC), gold(III) chloride hydrate $\left(\mathrm{HAuCl}_{4} \cdot x \mathrm{H}_{2} \mathrm{O}, 99.999 \%\right)$, hydroquinone ( $\left.\geq 99 \%\right)$, O-(2carboxyethyl)-O'-(2-mercaptoethyl)heptaethylene glycol (>95\%; $\mathrm{MW}=458.6 \mathrm{Da} ; 500 \mathrm{PEG})$, and $2100 \mathrm{Da}$ poly(ethylene glycol) 2-mercaptoethyl ether acetic acid (2000 PEG) were purchased from Merck Sigma-Aldrich (Darmstadt, Germany). 5000 Da alfa-carboxy-beta-mercaptoPEG (5000 PEG) was purchased from RAPP Polymere GmbH (Tübingen, Germany). The following cholesteryl-TEG modified DNA oligonucleotides were purchased from Eurogentec (Seraing, Belgium).

- 5'-TGG-ACA-TCA-GAA-ATA-AGG-CAC-GAC-GGACCC-3'-TEG-cholesterol (ssA)

- Cholesterol-TEG-5'-CCC-TCC-GTC-GTG-CCT-3' (ssB)

- 5'-TAT-TTC-TGA-TGT-CCA-AGC-CAC-GAGTTC-CCC-3'-TEG-cholesterol (ssC)

- Cholesterol-TEG-5' -CCC-GAA-CTC-GTG-GCT-3' (ssD)

Buffers. Four different buffers were used:

- $\mathrm{pH}(\mathrm{pD})=7.0,150 \mathrm{mM} \mathrm{NaCl}$, and $10 \mathrm{mM}$ Bis-Tris;

- $\mathrm{pH}(\mathrm{pD})=7.0,150 \mathrm{mM} \mathrm{NaCl}, 2.5 \mathrm{mM} \mathrm{CaCl}_{2}$, and 10 mM Bis-Tris;

- $\mathrm{pH}(\mathrm{pD})=4.0, \approx 3.8 \mathrm{mM}$ trisodium citrate dihydrate and $\approx 6.2 \mathrm{mM}$ citric acid monohydrate;

- PBS buffer $\mathrm{pH}(\mathrm{pD})=7.4,2.7 \mathrm{mM} \mathrm{KCl}, 137 \mathrm{mM} \mathrm{NaCl}$, and $10 \mathrm{mM}$ phosphate.

Buffers were prepared in

- $\mathrm{H}_{2} \mathrm{O}$ (Milli-Q purity with resistivity $\approx 18.2 \Omega \cdot \mathrm{cm}$; Merck Millipore, Molsheim, France);

- $100 \%, 80 \%, 60 \%$, and $40 \%$ of $\mathrm{D}_{2} \mathrm{O}$ in $\mathrm{H}_{2} \mathrm{O}$ corresponding to a molar fraction of $\mathrm{D}_{2} \mathrm{O}$, $x_{\mathrm{D}_{2} \mathrm{O}}=1.0,0.8,0.6$, and 0.4 , respectively;

- 4.55 wt \%, 3.80 wt \%, 2.99 wt \%, and 2.12 wt \% of glycerol in $\mathrm{H}_{2} \mathrm{O}$, which match the kinematic viscosity, $\nu$, of $100 \%, 80 \%, 60 \%$, and $40 \% \mathrm{D}_{2} \mathrm{O}$ solutions, respectively;

- 10.86 wt \% of glycerol in $\mathrm{H}_{2} \mathrm{O}$, which match the product of the density and viscosity, $\rho \eta$, of $100 \% \mathrm{D}_{2} \mathrm{O}$. 
The physical properties of all these solutions can be found in Table S1 in the Supporting Information.

The $\mathrm{pH}$ of buffers in $\mathrm{H}_{2} \mathrm{O}$ and glycerol/ $/ \mathrm{H}_{2} \mathrm{O}$ mixtures were determined using a Mettler-Toledo (Ohaio, US) $\mathrm{pH}$ meter. The $\mathrm{pH}(\mathrm{pD})$ for $\mathrm{D}_{2} \mathrm{O}$-containing buffers were determined using $\mathrm{pH}$ paper, the color of which was compared and matched against the color of a similar $\mathrm{pH}$ paper after submerging in the corresponding $\mathrm{H}_{2} \mathrm{O}$-based buffer. This procedure was followed to make sure that the buffers prepared in $\mathrm{H}_{2} \mathrm{O}, \mathrm{D}_{2} \mathrm{O}, \mathrm{D}_{2} \mathrm{O}$ / $\mathrm{H}_{2} \mathrm{O}$ mixtures, or glycerol $/ \mathrm{H}_{2} \mathrm{O}$ mixtures had the same $\mathrm{pH}$ (pD). Note that buffer dissociation constants depend on the chemical environment so the buffer will behave differently in $\mathrm{H}_{2} \mathrm{O}$ than in a glycerol/ $\mathrm{H}_{2} \mathrm{O}$ mixture. For this reason, adding equal amounts of the chemicals does not guarantee having the same $\mathrm{pH}$; it is therefore necessary to check and adjust the $\mathrm{pH}$ of each solution.

$\mathrm{H}_{2} \mathrm{O}$-based buffers were sterilized either by autoclaving at $120{ }^{\circ} \mathrm{C}$ for $20 \mathrm{~min}$ or by sterile filtration using $0.22 \mu \mathrm{m}$ Stericup-GV sterile vacuum filters (Millipore, France); all other buffers were sterile filtered using $0.22 \mu \mathrm{m}$ Stericup-GV sterile vacuum filters (Millipore, France). Note that the buffers filtered through the $0.22 \mu \mathrm{m}$ Stericup-GV sterile vacuum filters caused aggregation of the $\mathrm{SiO}_{2} \mathrm{NPs}$, suggesting that the filter membrane released leachate in the buffer. This problem was addressed by either prewashing the filters with buffer or autoclaving the buffers instead of filtering them; the latter was only done for $\mathrm{H}_{2} \mathrm{O}$-based buffers.

Gold Nanoparticles Synthesis. Gold nanoparticles (Au NPs) were synthesized and surface-modified with acidterminated poly(ethylene) glycol (PEG) with different molecular weights following the procedure outlined by Perrault and Chan. ${ }^{51} \mathrm{~A}$ seed solution of small Au NPs were made by quickly heating a $100 \mathrm{~mL}$ water solution containing $0.01 \%$ (w/ v) of $\mathrm{HAuCl}_{4}$. Once boiling, the solution was kept under vigorous stirring whereupon $3 \mathrm{~mL}$ of $1 \%(\mathrm{w} / \mathrm{v})$ sodium citrate solution was quickly added. The mixture was allowed to continue to boil for $10 \mathrm{~min}$ developing a ruby-red color. To make Au NPs with a diameter of approximately $100 \mathrm{~nm}$, a 100 $\mathrm{mL}$ water solution containing $0.025 \%(\mathrm{w} / \mathrm{v}) \mathrm{HAuCl}_{4}$ was mixed with $0.8 \mathrm{~mL}$ of seed solution. The mixture was rapidly stirred at room temperature whereupon $550 \mu \mathrm{L}$ of $1 \%(\mathrm{w} / \mathrm{v})$ sodium citrate solution was immediately added followed by 2.5 $\mathrm{mL}$ of a $30 \mathrm{mM}$ hydroquinone solution. The solution was continuously stirred for another $60 \mathrm{~min}$ until its color had stabilized, indicating that the reaction was completed. The size of the Au NPs was determined using AFM to be $\sim 70 \mathrm{~nm}$ in diameter. The $\mathrm{Au}$ NP concentration was calculated from the particle size and the amount of gold added to the synthesis assuming that all gold was consumed during the reaction.

$\mathrm{Au}$ NPs were surface modified by the reaction with acidterminated PEG-thiols with a molecular weight of $458.6 \mathrm{Da} O-$ (2-carboxyethyl)-O'-(2-mercaptoethyl)heptaethylene glycol, $2100 \mathrm{Da}$ poly(ethylene glycol) 2-mercaptoethyl ether acetic acid or $5000 \mathrm{Da}$ alfa-carboxy-beta-mercapto-PEG. On the basis of the $\mathrm{Au}$ NP size and concentration, the total surface area of the particles in the solution was calculated. Prior to surface modification, the Au NPs were concentrated 10 times by centrifugation $(1000 \mathrm{~g}, 20 \mathrm{~min})$ and dilution of the obtained pellet in Milli-Q water. The concentrated Au NPs were mixed with the different thiol-PEG molecules to reach a final concentration of approximately 10 PEG molecules per $\mathrm{nm}^{2}$ available $\mathrm{Au}$ NP surface area. The mixtures were incubated overnight whereupon the PEG-modfied $\mathrm{Au}$ NPs were separated from unbound thiol-PEG and salts by four repeated runs through centrifuge filter columns with $300 \mathrm{kDa}$ cutoff (PALL Nanosep Omega 300k). After the final washing step, $\mathrm{Au}$ NPs were kept as a concentrated stock solution until use.

POPC Vesicles Preparation. POPC was dissolved in chloroform and dried in a $50 \mathrm{~mL}$ round flask under vacuum at $60{ }^{\circ} \mathrm{C}$ using a rotavap setup. The dried lipids were left under vacuum overnight to get rid of any residual chloroform. The formed POPC lipid film was then rehydrated in $\mathrm{H}_{2} \mathrm{O}$-based buffer to a concentration of $1 \mathrm{mg} \cdot \mathrm{mL}^{-1}$, followed by a very brief bath sonication to dissolve any small traces of lipids off the walls of the flask. The POPC solution underwent five freeze/thawing cycles, and after which, the sample was extruded 31 times through $30 \mathrm{~nm}$ polycarbonate membranes (Whatman, UK) using a mini-extruder (Avanti, USA). The size of the vesicles was finally determined using dynamic light scattering (DLS) on Zetasizer Nano ZS (Malvern Panalytical, UK).

DNA Tethers Preparation. A 45-base pair DNA tether decorated with cholesterol anchors was prepared by mixing the different oligonucleotides (ssA, ssB, ssC, and ssD) as detailed hereafter. A total of $800 \mu \mathrm{L}$ of $10 \mu \mathrm{M}$ ssA was mixed with ssB $(\mathrm{AB})$, as well as ssC with ssD (CD). The mixtures were vortexed and left to hybridize for at least $30 \mathrm{~min}$ at $4{ }^{\circ} \mathrm{C}$. Afterward, the $A B$ and $C D$ segments were mixed together by vortexing and left to hybridize for at least $30 \mathrm{~min}$ at $4{ }^{\circ} \mathrm{C}$. The $\mathrm{ABCD}$ dsDNA was diluted in PBS to a final concentration of $0.5 \mu \mathrm{M}$.

QCM Experiments. The QCM experiments were conducted in an E4 system (Biolin, Sweden) coupled to a GX-274 autosampler (Gillson, USA) on AT-cut $5 \mathrm{MHz}$ quartz sensors, coated with $\mathrm{SiO}_{2}$. The sensors were either used without modification or after silanization with amine-terminated silanes. Bare sensors were cleaned by bath sonication for 15 min in 2 wt \%. SDS solution, followed by rinsing with Milli-Q water, then drying under an $\mathrm{N}_{2}$ flow, and finally treating with $\mathrm{O}_{2}$ plasma for 3 min directly before use. The temperature and flow rate were kept constant all through the QCM experiments at $25{ }^{\circ} \mathrm{C}$ and $50 \mu \mathrm{L} \cdot \mathrm{min}^{-1}$, respectively. At the end of the $\mathrm{Au}$ and $\mathrm{SiO}_{2} \mathrm{NPs}$ adsorption experiments, the solution in contact with the sensor was exchanged with ethanol $\geq 99.5 \%$, and then the sensors were dried in situ under an $\mathrm{N}_{2}$ flow and at a temperature of $60{ }^{\circ} \mathrm{C}$; finally, the temperature was lowered again to $25{ }^{\circ} \mathrm{C}$. This procedure was followed to dry the sensors for determining the response after drying (in air) and for further analysis using AFM and SEM while minimizing any drying effects, such as aggregation of the NPs on the surface. Step-by-step experimental and calculation procedures are presented in sections S3 and S4 in the Supporting Information.

Silanization of $\mathrm{SiO}_{2}$ Sensors. The sensors were first cleaned by bath sonication for $15 \mathrm{~min}$ in $2 \mathrm{wt} \%$ SDS solution, followed by rinsing with Milli-Q water, $\mathrm{O}_{2}$ plasma treatment for $3 \mathrm{~min}$, immersing the surface of the sensor for $30 \mathrm{~min}$ with $4 \mathrm{M} \mathrm{H}_{2} \mathrm{SO}_{4}$, thorough rinsing under flowing Milli-Q water, thorough rinsing with anhydrous methanol, and finally, drying under an $\mathrm{N}_{2}$ flow. The sensors were then incubated in $7.5 \mathrm{v} / \mathrm{v}$ $\%$ (3-aminopropyl)-dimethylethoxysilane in anhydrous methanol for at least $30 \mathrm{~min}$. Afterward, the sensors were rinsed thoroughly with anhydrous methanol to avoid precipitation of the silanes, which happens in the presence of water or ethanol. Finally, the sensors were rinsed with Milli-Q water and mounted in the instrument for use. 
Atomic Force Microscopy (AFM). A Bruker Dimension 3100 scanning probe microscopy system was used for ex-situ atomic force imaging of the surface of the dried QCM sensors. The height ( $z$-distance) of both $\mathrm{SiO}_{2}$ and Au NPs were used to determine their size. Detailed experimental and computation procedures are presented in section S5 in the Supporting Information.

Scanning Electron Microscopy (SEM). Scanning electron microscopy (Zeiss Ultra 55 FEG, in-lens mode, ETH $=2 \mathrm{kV}$ ) was used for general $e x$-situ characterization of the dried QCM sensor surfaces and for determination of NP surface coverage. Images were acquired at a location as close as possible to the center of the sensor; the area of the sensor which contributes the most to the QCM signal. We also tried to select an imaging area that did not show any visible drying effects, such as clear aggregation of the NPs. On the basis of the acquired images, NPs were counted using ImageJ; the total number of the NPs was then divided by the area of the image to obtain the number of NPs per $\mu \mathrm{m}^{2}$. Features much larger or much smaller than the size of the NPs were not counted toward the total number of the NPs. Features which showed a double layer of NPs were double counted. Examples of these different cases, as well as detailed experimental and computation procedures, are presented in section S5 in the Supporting Information.

Surface Resonance Plasmon (SPR) Experiments. The SPR experiments were conducted using a dual wavelength, 670 and $785 \mathrm{~nm}$, multiparametric SPR Navi420A (BioNavis, Finland) on silica-coated sensors. The sensors were cleaned by bath sonication for $15 \mathrm{~min}$ in $2 \mathrm{wt} \%$. SDS solution, followed by rinsing with Milli-Q water, then drying under an $\mathrm{N}_{2}$ flow, and finally treating in a UV/ozone chamber for 45 min directly before use. The temperature and flow rate were kept constant at $25{ }^{\circ} \mathrm{C}$ and $7 \mu \mathrm{L} \cdot \mathrm{min}^{-1}$, respectively.

\section{RESULTS AND DISCUSSION}

For QCM, as well as many mechanical resonators, the NavierStokes equation describing the coupled liquid can be reduced to one of the general textbook equations (see section S1 in the Supporting Information for detailed discussion),

$$
\frac{\partial}{\partial t}(\operatorname{curl} \mathbf{v})=\nu \Delta(\operatorname{curl} \mathbf{v})
$$

where $\mathbf{v}$ is the liquid velocity and $\nu \equiv \eta / \rho$ is the liquid kinematic viscosity ( $\eta$ and $\rho$ are the viscosity and density of the liquid). For firmly bound rigid adsorbates or for adsorbates with appreciably larger inertia than that of the adjacent liquid, the frequency induced by the dry mass of the adsorbate, $\Delta f^{\text {dry }}$, and the liquid-related change in frequency, $\Delta f^{\text {liq }}$, are additive. For such systems, the corresponding no-slip boundary condition at the surface- and adsorbate-liquid interface (i.e., the condition stating that the liquid velocity at the interface is the same as that of the sensor surface and adsorbate) depends only on the geometry of the interface. Accordingly, the solution of eq 2, $\mathbf{v}$, depends only on $\nu$ and the interfacial geometry. Further, the liquid-related change in frequency, $\Delta f^{\text {liq }}$, is proportional to the liquid-related force acting at the surface- and adsorbate-liquid interfaces. This force can be expressed via $\mathbf{v}$ and represented as a product of $\rho$ and a function, $F(\nu)$, which depends only on $\nu$ and the geometry. It can be done in general (section $S 1$ in the Supporting Information), without using an explicit expression for $\mathbf{v}$. In the context of QCM, $F(\nu)$ can be designated as $k_{f}$ which depends only on $\nu$ and the geometry as well; i.e., we have $\Delta f^{\text {liq }}=k_{f} \rho$, and thus, the measured frequency response, $\Delta f$, is given by

$$
\Delta f_{i}=\Delta f^{\mathrm{dry}}+\Delta f_{i}^{\mathrm{liq}}=\Delta f^{\mathrm{dry}}+k_{f} \cdot \rho_{i}
$$

where $i$ is a subscript introduced in order to specify the type of liquid in which the measurement is performed (compared to eq 1 , we do not indicate $n$ here). For the change in dissipation induced by a firmly bound adsorbate, by analogy, we obtain (section S1 in the Supporting Information)

$$
\Delta D_{i}=\Delta D^{\text {mech }}+\Delta D_{i}^{\text {liq }}=\Delta D^{\text {mech }}+k_{D} \cdot \eta_{i}
$$

where $\Delta D^{\text {mech }}$ is the term related to the mechanically dissipated energy, $\Delta D_{i}^{\mathrm{liq}}$ is the liquid-related term, and $k_{D}$ is the corresponding constant which depends on $\nu_{i}$ and the interfacial geometry.

Physically, $\Delta f^{\text {dry }}$ and $\Delta D^{\text {mech }}$ are associated with the motion of the adsorbate by itself, i.e., its motion in the absence of the surrounding liquid, with $k_{f} \rho_{i}$ and $k_{D} \cdot \eta_{i}$ being the NavierStokes-related liquid contributions. On the basis of the adsorbate system and liquids used, $\Delta f^{\text {dry }}$ and $\Delta D^{\text {mech }}$ might also include adsorbed liquid provided these liquids are not described by the Navier-Stokes equation. This distinction will be addressed in more detail when discussing the experimental results in the following sections.

If measurements are performed in one liquid alone, eqs 3 and 4 are not very helpful for practical applications because the dependence of $k_{f}$ and $k_{D}$ on $\nu$ and the interfacial geometry can be analytically expressed only for flat surfaces (e.g., eq 4 in ref 8). In principle, for all other cases, this dependence can be calculated numerically ${ }^{37,52}$ or analytically. ${ }^{53-56}$ Numerical estimations are, however, neither straightforward nor universal, whereas analytical expressions can practically be derived only by employing severe approximations, e.g., by using Brinkman's mean-field 1D equation as was previously done in order to describe the liquid-related response of the QCM for a rough surface $^{57}$ (section S1 in the Supporting Information) or some alternative approximations for specific geometries. ${ }^{53-56}$

Our strategy is different and generally applicable. In particular, we suggest to quantify $\Delta f^{\text {dry }}$ and $\Delta D^{\text {mech }}$ by determining $k_{f}$ and $k_{D}$ via measurements in different solutions matched with respect to their kinematic viscosity, i.e., in solutions with identical $\nu_{i}$ but different $\rho_{i}$ and $\eta_{i}$. According to eqs 3 and 4, this will allow us to exclude the contribution of coupled liquid to the QCM signal by extrapolating the measured values to $\rho=0$ and $\eta=0$, respectively, and thereby extract $\Delta f^{\text {dry }}$ and $\Delta D^{\text {mech }}$ from the respective intercepts, without requiring any information about the geometry of the adsorbate. Above, this approach has been validated for rigid adsorbate systems. Our experiments presented below show that it is applicable and remains fairly accurate even for viscoelastic adsorbate systems, such as biological soft matter.

To experimentally evaluate the applicability of the proposed approach, we have estimated the dry mass of $150 \mathrm{~nm} \mathrm{SiO}$ nanoparticles (NPs) electrostatically adsorbed on a flat QCM surface at coverages, $\theta$, ranging from 1 to $54 \%$. We also disentangled the liquid-related contribution to $\Delta D$ from the contribution associated with the attachment between the NPs and the surface. The latter was explored to gain new insights into the postulated influence of surface crowding of NPs on the stiffness of the attachment between each NP and the surface. ${ }^{52}$ 

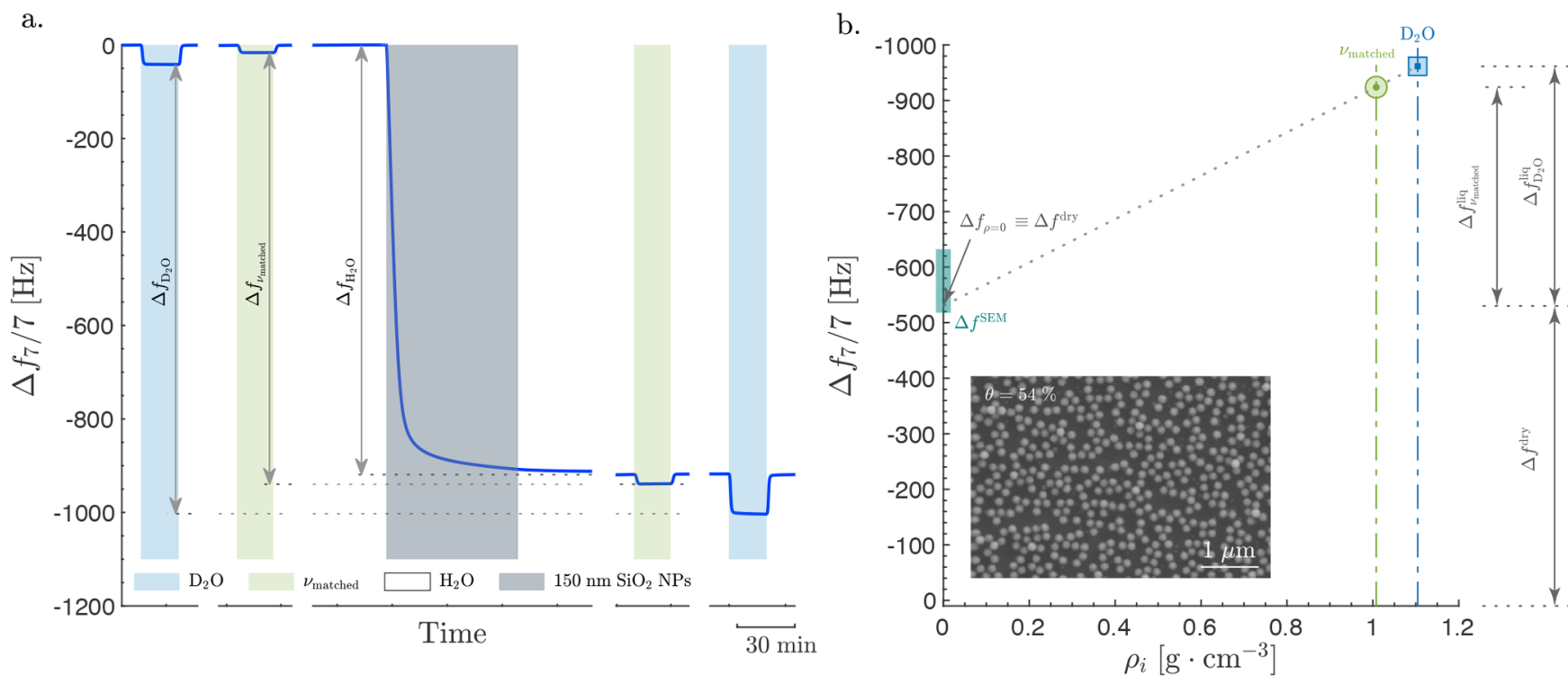

Figure 1. Experimental procedure for measuring the QCM frequency response in different solutions and disentangling the adsorbate mass and the coupled liquid mass. (a) The change in resonance frequency at the seventh overtone versus time for a flat poly-L-lysine (PLL)-coated substrate in contact with $\mathrm{H}_{2} \mathrm{O}, \mathrm{D}_{2} \mathrm{O}$, and a glycerol $/ \mathrm{H}_{2} \mathrm{O}$ mixture matching the kinematic viscosity of $\mathrm{D}_{2} \mathrm{O}, \nu_{\text {matched }}$ adsorption of $150 \mathrm{~nm} \mathrm{SiO} \mathrm{O}_{2}$ nanoparticles (NPs) until the jamming limit (corresponding to a surface coverage of 54\%) is reached in an $\mathrm{H}_{2} \mathrm{O}$-based buffer; exchanging the solutions again to $\nu_{\text {matched }}$ and $\mathrm{D}_{2} \mathrm{O}$. All solutions contained $10 \mathrm{mM}$ Bis-Tris, $\mathrm{pH}(\mathrm{pD})=7.0$, and $150 \mathrm{mM} \mathrm{NaCl}$. Detailed experimental and calculation procedures are available in sections S2-S4 and Figures S1-S4 in the Supporting Information. (b) Frequency responses in $\mathrm{D}_{2} \mathrm{O}$ and $\nu_{\text {matched }}$ versus liquid density and a linear extrapolation toward vanishing density. $\Delta f^{\mathrm{SEM}}$ represents the calculated frequency response based on the surface coverage determined using SEM; using a NPs size of $143 \mathrm{~nm}$ and a density of $2.0 \pm 0.2 \mathrm{~g} \cdot \mathrm{cm}^{-3}$. Inset shows an SEM image of the surface of the sensor after drying; scale bar $=1 \mu \mathrm{m}$. Detailed imaging and counting procedures for SEM are available in section S5 in the Supporting Information.

We also explored this approach for adsorption of carboxylated $70 \mathrm{~nm}$ gold $(\mathrm{Au}) \mathrm{NPs}$, as well as PEGylated Au NPs, to positively charged flat surfaces. For these experiments, we have varied the stiffness of the linker between the Au NPs and the surface by either varying the ionic strength of the solution or the length of the PEG linker. In addition, we tested the applicability of the approach on flexible biological NPs by assessing the dry mass of lipid vesicles tethered to the supported lipid bilayer (SLB) by DNA linkers. The similarity in structure and properties of lipid vesicles to exosomes and enveloped viruses makes them ideal models for assessing the applicability of the approach on biological nanoparticles. Finally, we extended the applicability of the approach beyond NPs by quantifying the dry mass of adsorbed humic acids (HAs), a highly hydrated ${ }^{58}$ and permeable ${ }^{59}$ natural polymeric material composed of supramolecular assemblies of relatively small molecules, ranging from $\simeq 200$ to $2500 \mathrm{Da}$ (ref 60) with key environmental importance, due to the role they play in, among others, transport of microbes, ${ }^{61}$ suppression of the release of greenhouse gases, ${ }^{62}$ and photochemical oxidation of pollutants. $^{63}$

Starting with high $\mathrm{SiO}_{2} \mathrm{NP}$ surface coverage $(\theta=54 \%)$, Figure 1a shows the three-step kinematic-viscosity-matching procedure in which $\mathrm{SiO}_{2} \mathrm{NPs}$ were electrostatically adsorbed to a smooth poly-L-lysine (PLL)-coated $\mathrm{SiO}_{2}$ sensor. After initial establishment of a baseline in buffered $\mathrm{H}_{2} \mathrm{O}$, the liquid in contact with the surface of the sensor was first replaced with buffered $\mathrm{D}_{2} \mathrm{O}$ followed by a buffered glycerol $/ \mathrm{H}_{2} \mathrm{O}$ mixture (4.55 wt \% glycerol) prepared to match the kinematic viscosity, $\nu=\eta / \rho$, of $\mathrm{D}_{2} \mathrm{O}$, henceforth referred to as $\nu_{\text {matched }}$. This was followed by adsorption of $\mathrm{SiO}_{2} \mathrm{NPs}$ in the $\mathrm{H}_{2} \mathrm{O}$-based buffer until the $\Delta f$ response plateaued, after which the $\nu_{\text {matched }}$ and $\mathrm{D}_{2} \mathrm{O}$ buffers were again subsequently injected into the flow cell. As indicated in Figure 1a, this procedure enables measurements of the response induced upon NP adsorption in different liquids, $\Delta f_{i}$.

A linear extrapolation of the frequency response in $\mathrm{D}_{2} \mathrm{O}$, $\Delta f_{\mathrm{D}_{2} \mathrm{O}}$, and the kinematic-viscosity-matched glycerol $/ \mathrm{H}_{2} \mathrm{O}$ mixture, $\Delta f_{\nu_{\text {matched }}}$, plotted versus $\rho_{i}$ (Figure 1b) yields an intercept at $\rho=0$ of $\Delta f_{\rho=0} \approx-530 \mathrm{~Hz}\left(\equiv 9540 \mathrm{ng} \cdot \mathrm{cm}^{-2}\right)$, a value which according to eq 3 represents the frequency shift of the dry mass of the adsorbed NPs, $\Delta f^{\text {dry }}$. The obtained value is in excellent agreement with the independently determined dry mass of $10350 \pm 1035 \mathrm{ng} \cdot \mathrm{cm}^{-2}$, based on ex-situ SEM images obtained after drying the sensor under nitrogen flow (Figure $1 b$, inset). Note that the liquid contribution to $\Delta f_{\mathrm{H}_{2} \mathrm{O}}$, representing the error introduced if $\Delta f$ alone is used to estimate the dry mass of the adsorbate, is appreciable $(\approx-390$ $\mathrm{Hz}$ equivalent to $>40 \%$ of the measured response). Further, this separation of the contributions to the measured response shows that both dry mass of the adsorbate and the mass corresponding to dynamically coupled liquid can be quantified via self-sufficient measurements using mechanical resonators alone, thus resolving a severe limitation of the technique that has for a long time disqualified its use for quantitative massuptake analysis in liquid environments. Although in most practical situations, adsorption measurements are carried out as in this experiment, i.e., on a flat QCM crystal already immersed in a liquid, we have, additionally, verified the applicability of the method for measurements conducted with reference to air as well as for flat surfaces without adsorbates. For flat surfaces without adsorbates, the extrapolation to $\rho=0$ should yield $\Delta f_{\rho=0}=0$. Indeed, the results presented in section S6 in the Supporting Information show that measurements conducted with reference to air yield the same dry mass as for 
a.

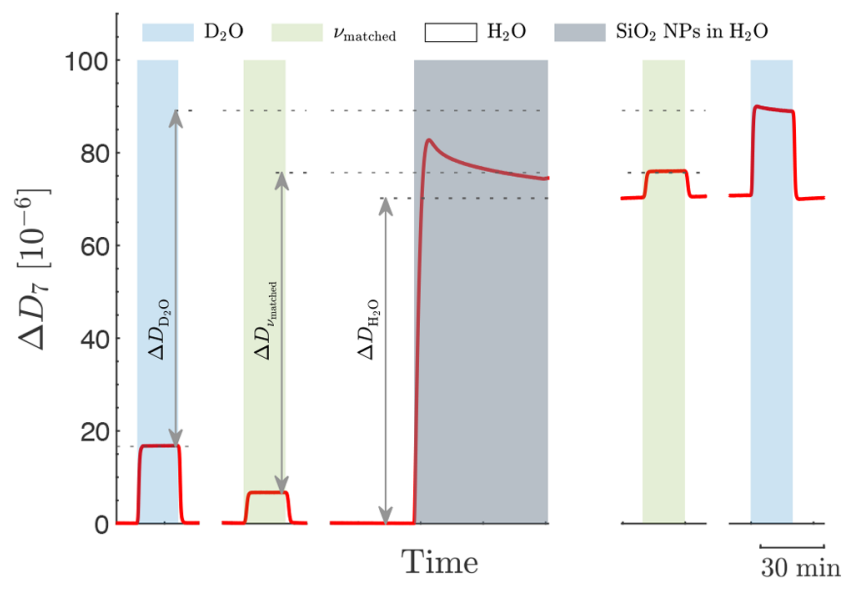

b.

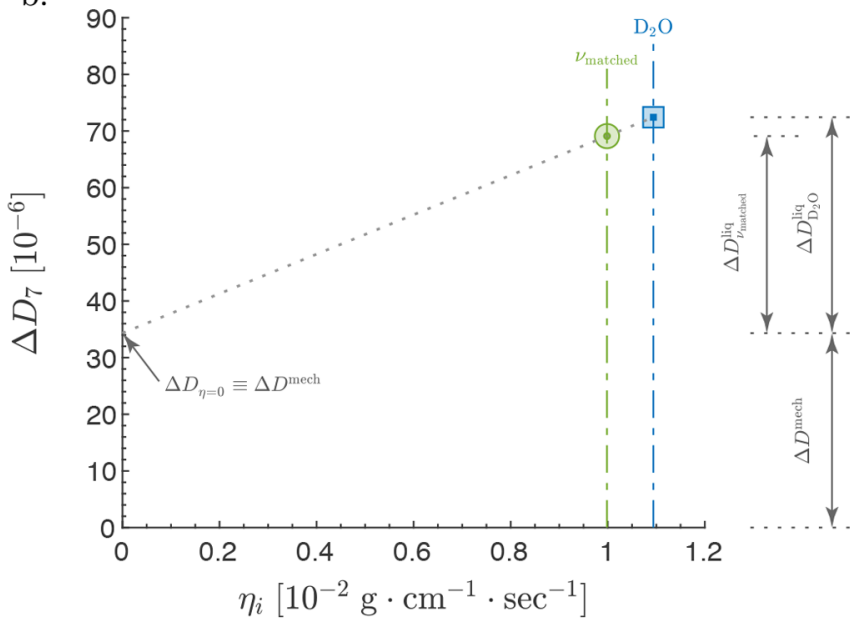

Figure 2. Experimental procedure for measuring the QCM dissipation response in different solutions and disentangling the mechanical and liquid contributions. (a) The change in dissipation at the seventh overtone versus time for a flat poly-L-lysine (PLL)-coated substrate in contact with $\mathrm{H}_{2} \mathrm{O}$, $\mathrm{D}_{2} \mathrm{O}$, and a glycerol $/ \mathrm{H}_{2} \mathrm{O}$ mixture matching the kinematic viscosity of $\mathrm{D}_{2} \mathrm{O}, \nu_{\text {matched }}$; the adsorption of $150 \mathrm{~nm} \mathrm{SiO} \mathrm{S}_{2}$ nanoparticles (NPs) until the jamming limit (corresponding to a surface coverage of 54\%) is reached in an $\mathrm{H}_{2} \mathrm{O}$-based buffer; exchanging the solutions again to $\nu_{\text {matched }}$ and $\mathrm{D}_{2} \mathrm{O}$. All solutions contained $10 \mathrm{mM}$ Bis-Tris, $\mathrm{pH}(\mathrm{pD})=7.0$, and $150 \mathrm{mM} \mathrm{NaCl}$. A detailed experimental and calculation procedure is available in sections S2-S4 and Figures S1-S4 in the Supporting Information. (b) Dissipation responses in $\mathrm{D}_{2} \mathrm{O}$ and $\nu_{\text {matched }}$ versus liquid viscosity and a linear extrapolation toward vanishing viscosity.

measurements conducted with the QCM crystal already immersed in a liquid; it also shows that for surfaces without adsorbates the extrapolated results to $\rho=0$ yields $\Delta f_{\rho=0} \approx 0$ (at all overtones), offering additional experimental verification to the kinematic-viscosity-matching approach and also providing an internal verification for the accuracy of the preparation of the kinematic-viscosity-matched solutions by rendering an intercept at $\Delta f_{\rho=0}=0$. As discussed in details in forthcoming sections, the extrapolation approach has been further verified using four different $\mathrm{D}_{2} \mathrm{O} / \mathrm{H}_{2} \mathrm{O}$ mixtures and their corresponding kinematic-viscosity-matched glycerol $/ \mathrm{H}_{2} \mathrm{O}$ mixtures.

In the analysis above, the adsorbed NPs are assumed to be (i) rigid and (ii) firmly linked to the surface; i.e., the measured $\Delta D_{i}$ should be solely due to the liquid contribution, $\Delta D_{i}^{\text {liq }}$, with no mechanically dissipated energy, $\Delta D^{\text {mech }}=0$. For the $\mathrm{SiO}_{2}$ NPs under consideration, assumption (i) is valid. Concerning assumption (ii), the corresponding contribution to the dissipation, $\Delta D^{\text {mech }}$, should be zero in order for the proposed approach to be strictly valid (see eq 4). It is therefore instructive to inspect the corresponding changes in $\Delta D$ (Figure 2a) for the same sequence of injection events shown in Figure 1a.

Extrapolation of $\Delta D_{\mathrm{D}_{2} \mathrm{O}}$ and the kinematic-viscosity-matched glycerol $/ \mathrm{H}_{2} \mathrm{O}$ mixture, $\Delta D_{\nu_{\text {mathed }}}$ to $\eta=0$ has an intercept $\Delta D_{\eta=0} \approx 34 \times 10^{-6}$ (Figure $2 \mathrm{~b}$ ) which is equivalent to $-\Delta D_{\eta=0} / \Delta f_{\rho=0}=0.06 \mathrm{~Hz}^{-1}$ or $10^{-14}$ per NP and corresponds to $\sim 50 \%$ of the measured $\Delta D_{\mathrm{H}_{2} \mathrm{O}}$. The nonzero positive $\Delta D_{\eta=0}$ was further confirmed by measurement conducted with reference to air as well as measurements of flat surfaces without adsorbate, the latter yielding $\Delta D_{\eta=0} \approx 0$ (section S6 in the Supporting Information). While $\Delta D_{\eta=0}=0$ for the NPs would have been unequivocal evidence of rigidly adsorbed NPs, i.e., negligible viscoelastic contribution to the QCM response, this result shows that there is indeed a measurable mechanical contribution to the energy dissipation from the adsorbed NPs. For adsorbate systems with measurable viscoelastic character, $\Delta D_{\eta=0}$ does not represent the dissipated energy under dry conditions, as in the case of perfectly rigid systems, but rather represents the mechanically dissipated energy in the adsorbate system surrounded by a liquid after excluding the energy dissipated in the liquid itself from the total dissipation response. The energy dissipated in the liquid is due to the corresponding viscous friction, which is referred to as $\Delta D^{\text {liq }}$ in this work. As a matter of fact, the dissipated energy under experimentally dry conditions, i.e., air, is in almost all cases negligible (Figure S5). $\Delta D_{\eta=0}$ in the case of rigid NPs is, thus, likely to reflect the mechanical energy dissipated due to the rotation and slippage of the NPs. ${ }^{52,64}$ Yet, the good agreement between the independent mass determination using SEM and the dry mass obtained by extrapolating $\Delta f_{i}$ in solutions matched with respect to the kinematic viscosity (Figure $1 \mathrm{~b}$ ) suggests that the extrapolation approach is still applicable for mass determination in spite of the positive $\Delta D_{\eta=0}$. In other words, the mechanical properties of the attachment between the NPs and the surface, reflected in a nonzero $\Delta D_{\eta=0}$, appears to have a negligible effect on $\Delta f_{\rho=0}$, thus suggesting that the kinematic-viscosity-matching approach may be applicable beyond perfectly rigid systems. We, therefore, aimed to investigate whether it is possible, using the measured QCM response only, to directly validate if the mechanical properties of the contact between a specific adsorbate and the surface have a significant influence on the dry mass determination or not.

To address this question, we performed experiments in which the stiffness of the attachment between NPs and the underlying surface was deliberately varied by replacing the PLL-coated surfaces with a surface modified with aminosilane (APDMES). Binding of NPs to the surface modified with APDMES resulted in significantly higher $\Delta D / \Delta f$ ratio than the binding to PLL did (Figure 3a), being indicative of weaker NPsurface interactions for the APDMES-modified surface (see section S7 of the Supporting Information). Furthermore, values of $\Delta f_{\mathrm{H}_{2} \mathrm{O}}$ had a more pronounced overtone dependence for the APDMES-modified surface than for the PLL-coated 

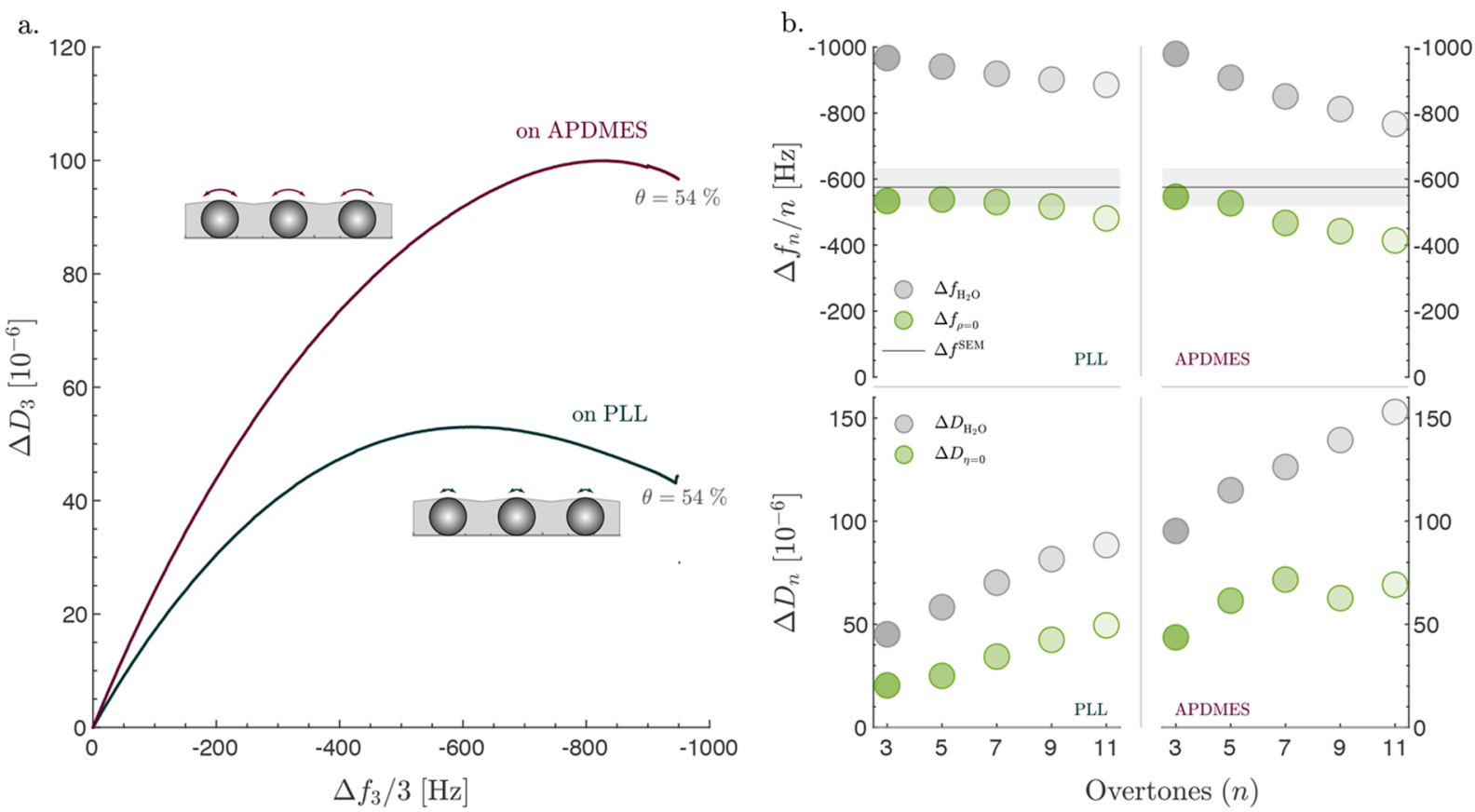

Figure 3. Effect of the flexibility of the attachment between the NPs and the substrate on dry mass determination. (a) Measured dissipation versus measured frequency at the third overtone for adsorption of $150 \mathrm{~nm} \mathrm{SiO}{ }_{2} \mathrm{NPs}$ on poly-L-lysine (PLL)-coated and on aminosilane (APDMES)modified sensors. Two inset schematics illustrate a stronger rocking motion (represented by longer arrow on the top of each NP) for the NPs adsorbed to APDMES- than PLL-modified sensors. (b) Overtone dependence for the two experiments exhibited in panel (a), showing the measured frequency and dissipation responses in $\mathrm{H}_{2} \mathrm{O}\left(\Delta f_{\mathrm{H}_{2} \mathrm{O}}\right.$ and $\left.\Delta D_{\mathrm{H}_{2} \mathrm{O}}\right)$ and the extrapolated frequency and dissipation responses $\left(\Delta f_{\rho=0}\right.$ and $\left.\Delta D_{\eta=0}\right) . \Delta f^{\mathrm{SEM}}$ represents the calculated frequency response based on the surface coverage determined using SEM imaging; utilizing a NPs size of $143 \mathrm{~nm}$ and density of $2.0 \pm 0.2 \mathrm{~g} \cdot \mathrm{cm}^{-3}$. The shaded area represents the uncertainty in the $\Delta f{ }^{\mathrm{SEM}}$ which arises from the uncertainty in the density of the NPs. All solutions contained $10 \mathrm{mM}$ Bis-Tris, $\mathrm{pH}(\mathrm{pD})=7.0$, and $150 \mathrm{mM} \mathrm{NaCl}$.

surface (Figure 3b, gray markers). Notably, while the overtone dependence essentially disappeared for the extrapolated $\Delta f_{\rho=0}$ for NPs bound to the PLL-coated surface, a clear overtone dependence was observed for NPs bound to the APDMESmodified surface (Figure $3 \mathrm{~b}$, green markers). This is expected since the adsorbate-induced $|\Delta f|$ typically decreases with increasing overtone number, $n$. This phenomenon arises from two different sources. The first is a viscoelastic contribution (referred to as softness in some previous work), which results in decreasing $|\Delta f|$ with increasing $n .^{65}$ The second is from an $n$-dependent liquid response; i.e., $|\Delta f|$ also decreases with increasing $n$. Notably, the $n$-dependent liquid response will also occur for rigidly coupled adsorbates; this has been previously shown through theoretical models ${ }^{65}$ as well as through experimental results of rough electrodeposited copper adlayers. ${ }^{66,67}$ Hence, for systems with a negligible viscoelastic influence on $\Delta f$, the overtone dependence of $\Delta f$ will thus arise primarily from the liquid contribution, and therefore the overtone dependence is expected to vanish if the liquid contribution is eliminated using the extrapolation approach. The observed difference in the overtone dependence between APDMES and PLL for $\Delta f_{\rho=0}$ can thus be attributed to a higher viscoelastic contribution upon NP adsorption on APDMESmodified than upon PLL-coated surfaces. This conclusion is further supported by the higher $\Delta D_{\mathrm{H}_{2} \mathrm{O}}$ and $\Delta D_{\eta=0}$ values measured for the APDMES-coated surface where both the value of $\Delta D_{\eta=0}$ and the deviation between $\Delta f_{\rho=0}$ and the dry mass determined using SEM decrease with decreasing $n$ (Figure $3 \mathrm{~b}$ ), suggesting a weaker contribution from viscoelastic losses to $\Delta f_{\rho=0}$ at lower $n$. In fact, the dry mass of NPs adsorbed to the APDMES-modified surface determined from $\Delta f_{\rho=0}$ at $n=3$ agreed perfectly with the SEM-determined value, within the uncertainty limits of the SEM approach. The overtone dependence on the linker stiffness has been further verified using two sets of experiments: (i) carboxylated Au NPs on APDMES at different ionic strengths (Figure S13) and (ii) PEGylated $\mathrm{Au}$ NPs with different PEG linker lengths on APDMES (Figure S13). These experiments clearly demonstrate the increasing overtone dependence of the measured and extrapolated frequency response with decreasing linker stiffness. It also shows that for all the experiments, even for $\mathrm{Au}$ NPs with 5000 Da PEG linker, the extrapolated frequency response at $n=3$ accurately estimates the dry mass of the NPs within less than $10 \%$ error compared to the complementary measurements of QCM in air.

An additional long-standing question is to what extent QCM data reflect lateral interactions between closely packed adsorbates on a surface. In particular, as seen in Figures 2a and $3 a, \Delta D$ reaches a transient maximum (peak) and starts decreasing again as more NPs adsorb. This transient peak has been previously ascribed to hydrodynamic stabilization of NPs, i.e., less rotation and slippage, that becomes appreciable when NPs are sufficiently close to each other, ${ }^{52}$ i.e., at high-enough surface coverage. We were thus curious to inspect whether the kinematic-viscosity-matching approach applied for a varying surface coverage $(\theta)$ of NPs could contribute new insight into this issue. Both the dry mass of NPs, $\Delta m_{\rho=0}$, and the mechanically dissipated energy associated with the contact zone between NPs and the surface, $\Delta D_{\eta=0}$, were therefore determined at various surface coverages, $\theta$, ranging from 1 to $54 \%$, (Figure $4 \mathrm{a}, \mathrm{c}$ ). The mass determined using the 

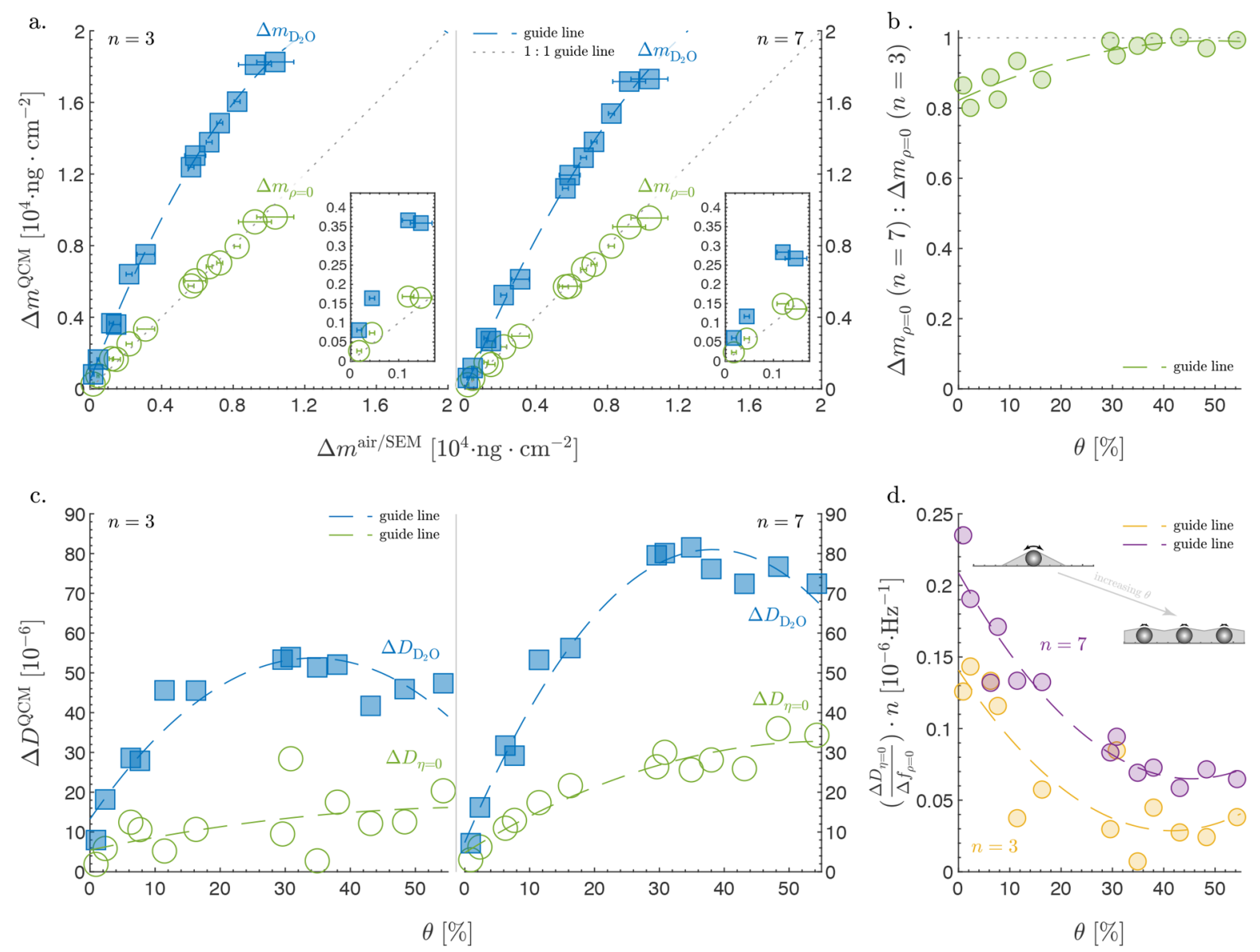

Figure 4. Disentangling the liquid and adsorbate mass/mechanical contributions to the QCM response of adsorbed $\mathrm{SiO}_{2}$ nanoparticles over a broad range of surface coverages. (a) Comparison between the measured QCM mass in $\mathrm{D}_{2} \mathrm{O}, \Delta m_{\mathrm{D}_{2} \mathrm{O}}$, and the mass obtained by extrapolating the responses in $\mathrm{D}_{2} \mathrm{O}$ and in a glycerol $/ \mathrm{H}_{2} \mathrm{O}$ mixture matching the kinematic viscosity of $\mathrm{D}_{2} \mathrm{O}, \nu_{\text {matched }}$, to $\rho=0, \Delta m_{\rho=0}$, versus the mass determined using SEM imaging or frequency response in air. Error bars represent the uncertainty in the air or SEM measurements as detailed in section S5 in the Supporting Information. The QCM mass was calculated using the Sauerbrey equation for $5 \mathrm{MHz}$ sensors: $\Delta m=-18.0 \cdot \Delta f$. Results are shown for the third and seventh overtone $(n=3$ and 7$)$; results from all overtones are presented in Figure S16. Insets show a zoomed-in portion of the plot in the low adsorbed mass region. (b) Ratio of the mass determined using the results of the seventh overtone to that of the third overtone versus surface coverage, $\theta$. The surface coverages were calculated based on the SEM images or the QCM response in air. (c) Comparison between the measured QCM energy dissipation in $\mathrm{D}_{2} \mathrm{O}, \Delta D_{\mathrm{D}_{2} \mathrm{O}}$, and the mechanically dissipated energy obtained by extrapolating the responses in $\mathrm{D}_{2} \mathrm{O}$ and in $\nu_{\text {matched }}$ to $\eta=0, \Delta D_{\eta=0}$, versus the surface coverage. Results are shown for the third and seventh overtone; results from all overtones are given in Figure S17. (d) Mechanical energy dissipated normalized by the frequency response of the dry mass for the NPs versus surface coverage at the third and seventh overtone. Inset shows a schematic illustrating a weaker rocking motion and thus less mechanically dissipated energy per particle at high surface coverage than low surface coverage. The length of the arrows on the top of NPs represents the strength of the rocking motion, which reflects the mechanically dissipated energy. All solutions contained $10 \mathrm{mM}$ Bis-Tris, $\mathrm{pH}(\mathrm{pD})=7$, and $150 \mathrm{mM} \mathrm{NaCl}$.

extrapolation approach, $\Delta m_{\rho=0}$ calculated based on $\Delta f_{\rho=0}$ using eq 1 , and the mass determined using QCM in air or SEM, $\Delta m^{\text {air/SEM }}$, are indeed in almost perfect agreement over the entire coverage regime (Figure $4 \mathrm{a}$ ). The overtone dependence of $\Delta m_{\rho=0}$ was fairly weak: the ratio between the seventh to the third overtone values of $\Delta m_{\rho=0}$ ranged from $\sim 80 \%$ at the lowest coverage to higher than $95 \%$ above $30 \%$ coverage (Figure 4a,b), demonstrating that the kinematic-viscosity approach can be used to successfully disentangle the dry and liquid mass in a broad coverage regime. Further, the transient peak in the measured energy dissipation, $\Delta D_{\mathrm{D}_{2} \mathrm{O}}$, disappears in the corresponding curve for the extrapolated $\Delta D_{\eta=0}$ (Figure $4 c)$, revealing that the changes in liquid-related energy dissipation versus surface coverage is the source of the observed transient peak in the measured dissipation. We also observed that $\Delta D_{\eta=0}$ contributes only between 30 and $50 \%$ to the measured dissipation at all surface coverages; i.e., the larger part of the dissipated energy is liquid-related in nature. Additionally, the contribution from the mechanically dissipated energy normalized by the extrapolated frequency response, $\Delta D_{\eta=0} / \Delta f_{\rho=0}$, decreases with increasing $\theta$ (Figure $4 \mathrm{~d}$ ), demonstrating that the mechanical energy dissipated per NP decreases with increasing $\theta$. These observations show that the NPs are indeed mechanically stabilized with increasing $\theta$, in good agreement with previous finite element modeling (FEM) of similar systems, ${ }^{52}$ a conclusion also confirmed by the observed decrease in overtone dependence of $\Delta f_{\rho=0}$ with increasing $\theta$ (Figure $4 \mathrm{~b}$ ). This analysis thus shows that the kinematic-viscosity-matching approach can be used not only to accurately determine the dry mass of the adsorbate over a broad coverage range but also to experimentally quantify the mechanically dissipated energy, opening up an new avenue to access various material and/or linker properties. 
a.

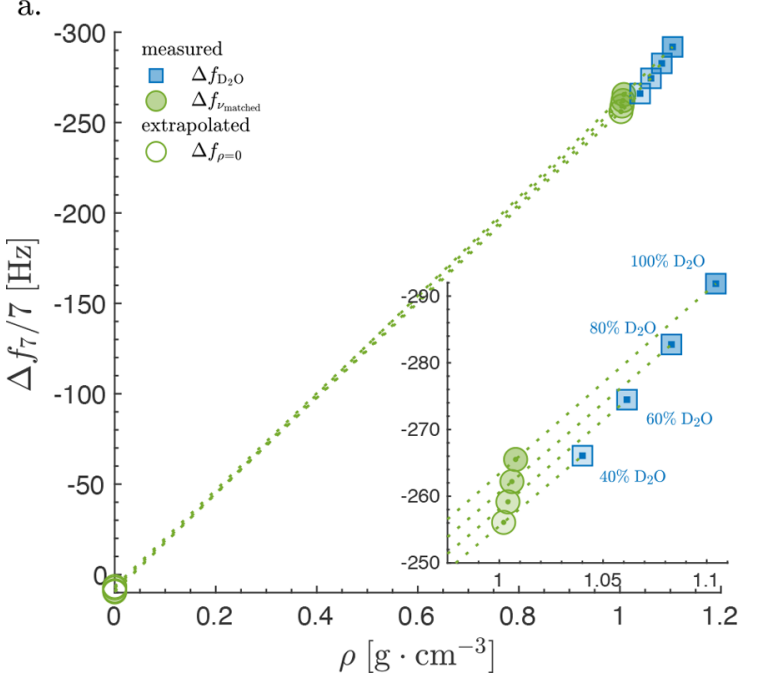

b.

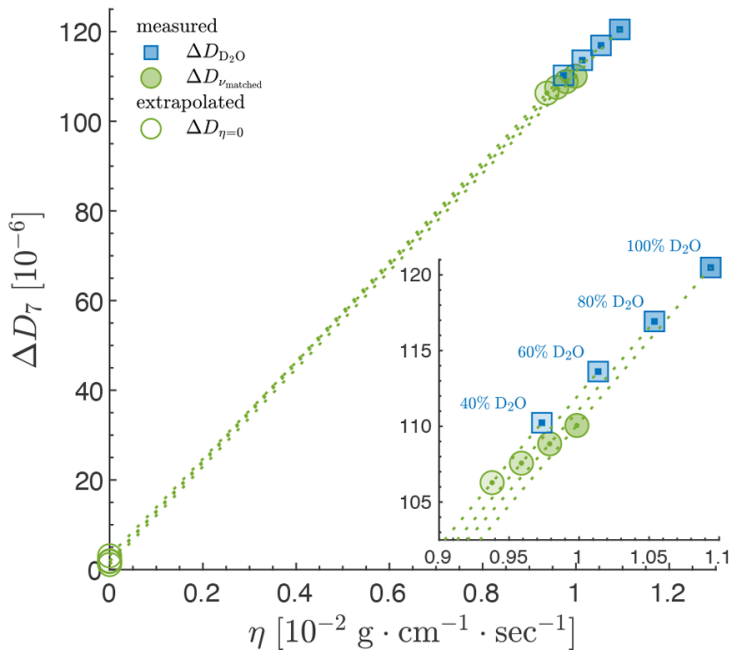

Figure 5. Extrapolation from different $\mathrm{D}_{2} \mathrm{O} / \mathrm{H}_{2} \mathrm{O}$ mixtures and their kinematic-viscosity-matched glycerol/ $\mathrm{H}_{2} \mathrm{O}$ mixtures. (a) Frequency versus liquid density and (b) dissipation versus liquid viscosity of four different $\mathrm{D}_{2} \mathrm{O} / \mathrm{H}_{2} \mathrm{O}$ mixtures and their kinematic-viscosity-matched glycerol/ $\mathrm{H}_{2} \mathrm{O}$ mixtures. The responses represent exchanging the medium in contact with a flat substrate from air to the different liquids. Shown as well are the extrapolation lines to $\rho=0$ and $\eta=0.100 \%, 80 \%, 60 \%$, and $40 \% \mathrm{D}_{2} \mathrm{O}$ correspond to the molar fractions $1.0,0.8,0.6$, and 0.4 of $\mathrm{D}_{2} \mathrm{O}$. Results were obtained from measurements on an aminosilane-modified sensor using a citrate-based buffer, $\mathrm{pH}(\mathrm{pD})=4.0$. Average results from 10 replicas and the corresponding standard deviations are shown in Figures S11 and S18 in the Supporting Information.

So far, we have relied on complementary methods, i.e., QCM in air and SEM, to verify the accuracy of the extrapolation approach. The objective of this work is to obtain accurate, trustworthy results while obviating the need for any complementary methods. As for any other scientific method, obtaining reliable data can only be achieved by conducting replicas and assessing the variability from one measurement to another. However, in some cases, it is very challenging to reproduce the same exact experimental conditions and thus outcome, such as for example, the same exact surface coverage of an adsorbate. By using more than one $\mathrm{D}_{2} \mathrm{O} / \mathrm{H}_{2} \mathrm{O}$ mixture and their matched $\mathrm{H}_{2} \mathrm{O}$ /glycerol mixture to estimate the dry mass and mechanical properties, one can extend the kinematicviscosity-matching approach to provide additional confidence to the results obtained from a single measurement. Figure 5 shows the responses measured upon exchanging the medium in contact with a flat substrate from air to four different $\mathrm{D}_{2} \mathrm{O}$ / $\mathrm{H}_{2} \mathrm{O}$ mixtures and their corresponding kinematic-viscositymatched glycerol $/ \mathrm{H}_{2} \mathrm{O}$ mixtures. Since there is no adsorption taking place in this process, the dry mass and the mechanical dissipated energy should be zero. Indeed, Figure 5 shows that the extrapolation from the four different pairs of kinematicviscosity-matched solutions yields $\Delta f_{\rho=0} \approx 0$ and $\Delta D_{\eta=0} \approx 0$. We have applied this approach for flat substrates without adsorbate (Figures 5 and S18), $150 \mathrm{~nm} \mathrm{SiO} 2$ NPs at different surface coverages (Figure S19), $70 \mathrm{~nm} \mathrm{Au} \mathrm{NPs} \mathrm{at} \mathrm{different}$ ionic strengths (Figure S20), and $70 \mathrm{~nm}$ PEGylated Au NPs with different PEG linker lengths (Figure S21). The results acquired from all these experiments show that $\Delta f_{\rho=0}$ and $\Delta D_{\eta=0}$, obtained by extrapolation of the response in four different $\mathrm{D}_{2} \mathrm{O} / \mathrm{H}_{2} \mathrm{O}$ mixtures with their corresponding kinematic-viscosity-matched glycerol $/ \mathrm{H}_{2} \mathrm{O}$ mixtures to $\rho=0$ and $\eta$ $=0$, all converge to similar values. The results showed systematic dependence for neither $\Delta f_{\rho=0}$ nor $\Delta D_{\eta=0}$ on the $\mathrm{D}_{2} \mathrm{O}$ to $\mathrm{H}_{2} \mathrm{O}$ mixing ratios. These results provide further verification for the kinematic-viscosity-matching approach to determine the adsorbate mass and the mechanical properties of the adsorbate/surface interactions and verify the accuracy of
$\Delta f_{\rho=0}$ to determine the adsorbate mass, in agreement with the evidence provided based on the results from QCM in air and SEM imaging. This is a more crucial control in the case of $\Delta D_{\eta=0}$, because there is limited existing knowledge with respect to the nature of the mechanically dissipated energy and whether it is affected by the properties of the liquid. If there were a dependency on the liquid properties, one would have expected a systematic increase or decrease in $\Delta D_{\eta=0}$ with the increasing $\mathrm{D}_{2} \mathrm{O}$ concentrations in the $\mathrm{D}_{2} \mathrm{O} / \mathrm{H}_{2} \mathrm{O}$ mixtures; these results suggest that the mechanically dissipated energy was not affected by the different liquid properties within the range of liquids used in this work. Additionally, the possibility to determine $\Delta f_{\rho=0}$ and $\Delta D_{\eta=0}$ based on the extrapolation of four couples of data points provides a robust statistical verification of the analysis, especially for systems with small $\Delta f$ and $\Delta D$ responses. Note that in this work we determine the dry mass and mechanically dissipated energy at down to $\theta=$ $1 \%$ of $150 \mathrm{~nm} \mathrm{SiO}{ }_{2} \mathrm{NPs}$, which is equivalent to $\Delta f_{\rho=0} \approx-15$ $\mathrm{Hz}$ and $\Delta D_{\eta=0} \approx 2 \times 10^{-6} ;-15 \mathrm{~Hz}$ corresponds to the mass of monolayer of small protein of $\sim 5 \mathrm{~nm}$ in diameter at the jamming limit $(\theta=54 \%)$. Finally, it is also worth noting that one could achieve a sound statistical verification of $\Delta f_{\rho=0}$ and $\Delta D_{\eta=0}$, as the one achieved using different $\mathrm{D}_{2} \mathrm{O} / \mathrm{H}_{2} \mathrm{O}$ mixtures, by exchanging the same kinematic-viscosity-matched liquids, e.g., $\mathrm{D}_{2} \mathrm{O}$ and 4.55 wt \% glycerol in $\mathrm{H}_{2} \mathrm{O}$, several times before and after the adsorbate.

Encouraged by the results obtained using the kinematicviscosity-matching approach applied on rigid NPs even under weak attachment conditions, we decided to explore the applicability of the approach to less rigid, biological NPs. As a proof of concept, we explored the possibility to determine the dry mass of POPC vesicles anchored to a supported lipid bilayer (SLB) via DNA tethers (Figure 6a,d). As previously described, ${ }^{68}$ DNA tethers were formed of 45 base pairs with a stretched length of $\sim 15 \mathrm{~nm}$ and have two cholesterol anchors at its ends enabling self-insertion of one end into the SLB and the other into the POPC vesicles. The flexibility of the DNA linkers and the POPC vesicles suggests that the system will 

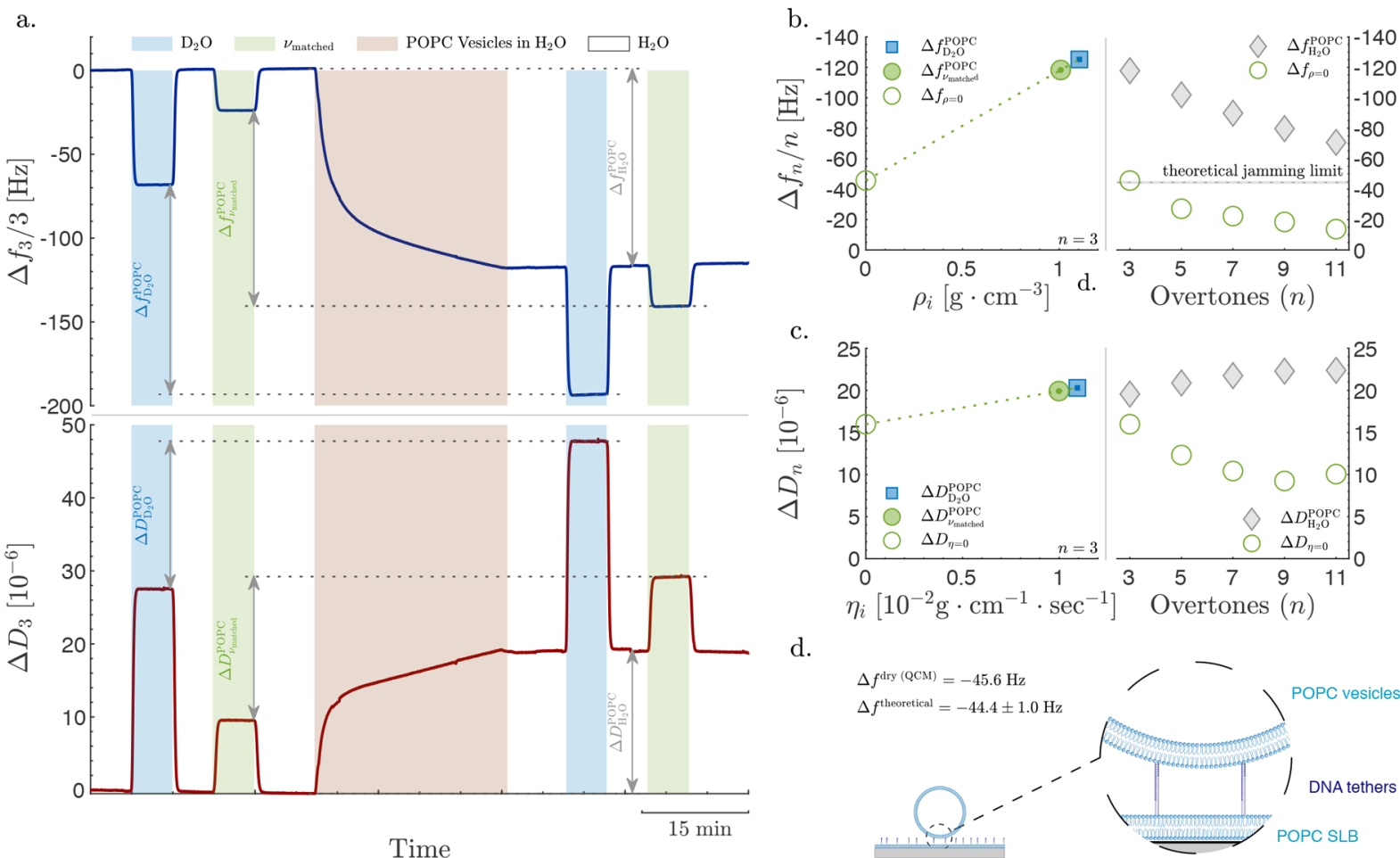

d.

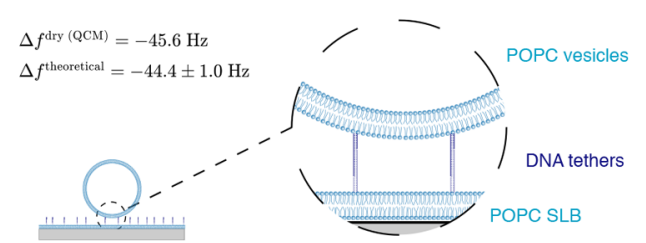

Figure 6. Disentangling the liquid and adsorbate mass/mechanical contributions to the QCM response of a tethered lipid vesicles. (a) The change in resonance frequency (upper panel) and dissipated energy (lower panel) at the third overtone versus time for the tethering of POPC lipid vesicles (size distribution shown in Figure S23) to a supported lipid bilayer (SLB) through DNA molecules with cholesterol anchors. The buffer was exchanged to a $\mathrm{D}_{2} \mathrm{O}$-based buffer and a glycerol/ $\mathrm{H}_{2} \mathrm{O}$ mixture matching the kinematic viscosity of $\mathrm{D}_{2} \mathrm{O}, \nu_{\text {matched }}$, before and after the POPC adsorption step. A complete experimental procedure including the formation of SLB and attachment of the DNA tethers is shown in Figure S23. (b) Frequency responses (left panel) for the POPC vesicles in $\mathrm{D}_{2} \mathrm{O}$ and $\nu_{\text {matched }}$ versus liquid density and a linear extrapolation toward vanishing density, $\rho=0$. Overtone dependence (right panel) for the measured frequency responses in $\mathrm{H}_{2} \mathrm{O}, \Delta f_{\mathrm{H}_{2} \mathrm{O}}$, and the extrapolated frequency responses, $\Delta f_{\rho=0}$, of the POPC vesicles. The theoretical frequency response, $\Delta f^{\text {theoretical }}$, was calculated using the Sauerbrey equation for $5 \mathrm{MHz}$ sensors: $\Delta m=$ $-18.0 \cdot \Delta f$ based on POPC molecular weight of $760.076 \mathrm{~g} \cdot \mathrm{mol}^{-1}$, area per lipid of $0.683 \pm 0.015 \mathrm{~nm}^{2}$ (ref 69 ) and assumed surface coverage of $\theta=$ $54 \%$. The shaded area represents the uncertainity in the theoretical estimation, arising from the uncertainity in the area per lipid molecule. (c) Dissipation responses (left panel) for the POPC vesicles from $\mathrm{D}_{2} \mathrm{O}$ and $\nu_{\text {matched }}$ versus liquid viscosity and a linear extrapolation toward vanishing viscosity, $\eta=0$. Overtone dependence (right panel) for the measured dissipation responses in $\mathrm{H}_{2} \mathrm{O}, \Delta D_{\mathrm{H}_{2} \mathrm{O}}$, and the extrapolated dissipation, $\Delta D_{\eta=0}$, of the POPC vesicles. (d) A schematic showing POPC vesicles tethered to SLB via DNA molecules. Experimental details, characterization of vesicles, and calculations are presented in section S11 in the Supporting Information. All solutions contained $10 \mathrm{mM}$ phosphate buffer, $\mathrm{pH}$ ( $\mathrm{pD}$ ) $=7.4,2.7 \mathrm{mM} \mathrm{KCl}$, and $137 \mathrm{mM} \mathrm{NaCl}$.

have an appreciable viscoelastic character; indeed, the measured $\Delta f_{\mathrm{H}_{2} \mathrm{O}}$ showed a very strong overtone dependence ranging from $-120 \mathrm{~Hz}$ at $n=3$ to $-70 \mathrm{~Hz}$ at $n=11$ (Figure $6 \mathrm{~b})$, and the $-\Delta D_{\mathrm{H}_{2} \mathrm{O}} / \Delta f_{\mathrm{H}_{2} \mathrm{O}}$ ratio ranged from 0.17 at $n=3$ to 0.32 for $n=11$ (Figure $6 \mathrm{~b}, \mathrm{c}$ ). Extrapolation of the frequency response in $\mathrm{D}_{2} \mathrm{O}$ and $\nu_{\text {matched }}$ buffers toward $\rho=0$ yields an intercept $\Delta f_{\rho=0}$ of $-45.6 \mathrm{~Hz}$ at $n=3$ but with a strong overtone dependence (Figure 6b). $\Delta f_{\rho=0}$ at $n=3$ is in excellent agreement with the theoretically estimated frequency response using the Sauerbrey equation (eq 1) based on the POPC molecular weight of $760.076 \mathrm{~g} \cdot \mathrm{mol}^{-1}$, area per lipid of $0.683 \pm$ $0.015 \mathrm{~nm}^{2}$ (ref 69), and surface coverage of $\theta=54 \%$. The assumed surface coverage is justified by the plateauing adsorption profile of the POPC vesicles (Figure 6a), which indicates that the surface coverage is very close to the jamming limit. Notably, the theoretical frequency response is independent of the size of the vesicles as detailed in section S11 in the Supporting Information. It is worth mentioning that $\Delta f_{\rho=0}$ determined here represents the dry mass of the lipids only in the POPC vesicles without the mass of the liquid in their core. This is because both $\mathrm{D}_{2} \mathrm{O}$ and glycerol are permeable to lipid membranes: ${ }^{70,71}$ with $\mathrm{D}_{2} \mathrm{O}$ and $\mathrm{H}_{2} \mathrm{O}$ having permeability coefficients in the range of $10^{-3}$ to $10^{-2} \mathrm{~cm} \cdot \mathrm{s}^{-1}$ and glycerol in the range of $10^{-6}$ to $10^{-5} \mathrm{~cm} \cdot \mathrm{s}^{-1}$; $^{71}$ thus, the liquid inside the vesicles is almost instantaneously exchanged when exchanging the bulk liquid. ${ }^{72}$ From a theoretical perspective, the liquid inside the vesicles is in this case included in the Navier-Stokes-related liquid contributions, i.e., $k_{f} \rho_{i}$ in eq 3. If one instead would use two liquids that are impermeable to the vesicles, $k_{f} \rho_{i}$ would not include the liquid inside the vesicles, and thus $\Delta f_{\rho=0}$ would represent the mass of the lipids and the liquid inside the vesicles-a distinction that is very important for correct data interpretation. Moreover, it is crucial to emphasize that determining the dry mass of the vesicles was only possible because the tethered lipid vesicles are expected to have the same structural, distribution, and binding properties in both $\mathrm{D}_{2} \mathrm{O}$ and $\nu_{\text {matched }}$ buffers; measurable changes in the properties of the adsorbate system when exchanging between the two liquids would have resulted in different $k_{f}$ and $k_{D}$ values for each of the liquids and thus an inaccurate estimation of $\Delta f_{\rho=0}$ and $\Delta D_{\eta=0}$. Finally, by 
a.

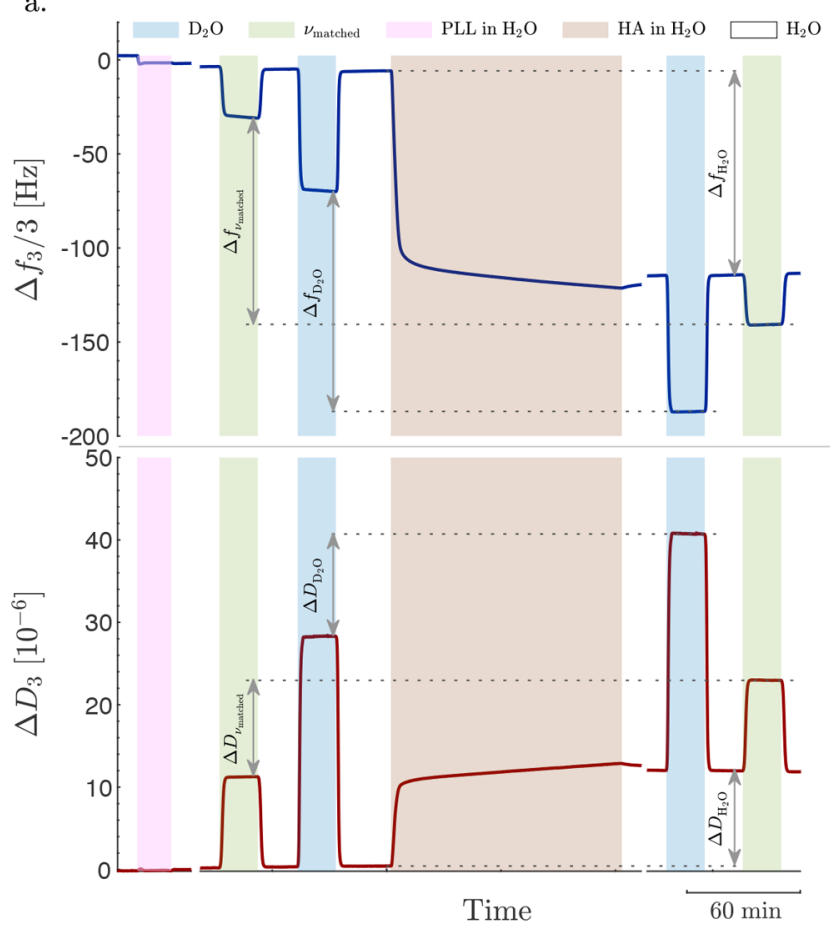

b.
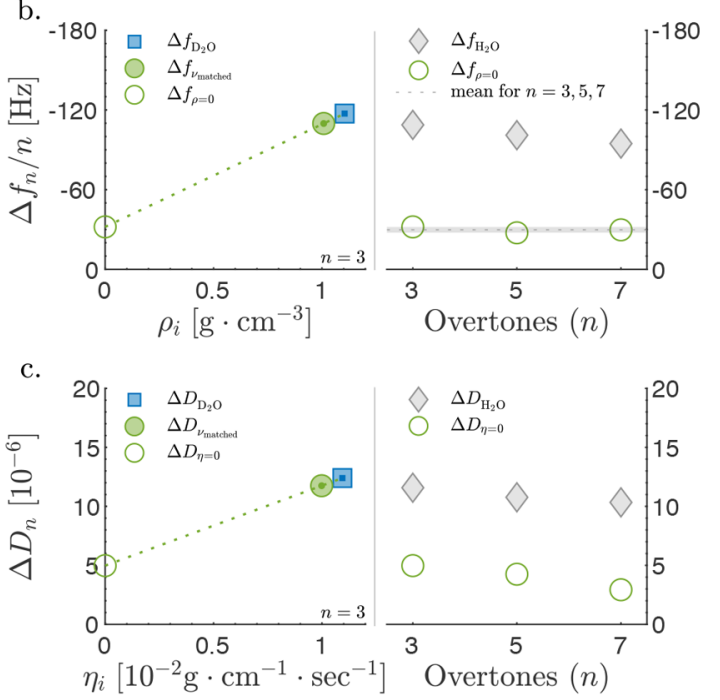

d.

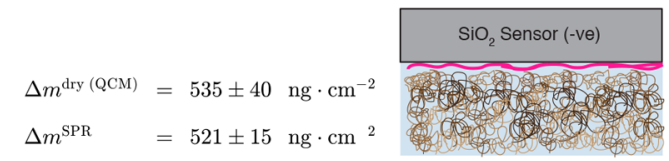

Figure 7. Disentangling the liquid and adsorbate mass/mechanical contributions to the QCM response of a polymer adlayer. (a) The change in resonance frequency (upper panel) and dissipated energy (lower panel) at the third overtone versus time for the adsorption of poly-L-lysine (PLL) adlayer on a bare $\mathrm{SiO}_{2}$ sensor, followed by deposition of a humic acids (HA) adlayer. The buffer was exchanged to $\mathrm{D}_{2} \mathrm{O}$-based buffer and glycerol/ $\mathrm{H}_{2} \mathrm{O}$ mixture matching the kinematic viscosity of $\mathrm{D}_{2} \mathrm{O}, \nu_{\text {matched }}$, before and after the HA adsorption step. (b) Frequency responses (left panel) for the HA layers from $\mathrm{D}_{2} \mathrm{O}$ and $\nu_{\text {matched }}$ versus liquid density and a linear extrapolation toward vanishing density, $\rho=0$. Overtone dependence (right panel) for the measured frequency responses in $\mathrm{H}_{2} \mathrm{O}, \Delta f_{\mathrm{H}_{2} \mathrm{O}}$, and the extrapolated frequency responses, $\Delta f_{\rho=0}$, of the HA adlayer. Results are shown for $n=3,5$, and 7; results from higher overtone numbers could not be utilized due to unstable instrumental signal. The dashed line represents the mean from the three overtones, and the shaded area represents the standard deviation. (c) Dissipation responses (left panel) for the HA layers from $\mathrm{D}_{2} \mathrm{O}$ and $\nu_{\text {matched }}$ versus liquid viscosity and a linear extrapolation toward vanishing viscosity, $\eta=0$. Overtone dependence (right panel) for the measured dissipation responses in $\mathrm{H}_{2} \mathrm{O}, \Delta D_{\mathrm{H}_{2} \mathrm{O}}$, and the extrapolated dissipation, $\Delta D_{\eta=0}$, of the $\mathrm{HA}$ adlayer. Results are shown for $n=3$, 5, and 7; results from higher overtone numbers could not be utilized due to the unstable instrumental signal. (d) A schematic showing the HA adlayer (in brown) on the PLL (in magenta)-coated $\mathrm{SiO}_{2}$ sensor. The dry mass, $\Delta m^{\text {dry }}(\mathrm{QCM})$, was calculated using the Sauerbrey equation for $5 \mathrm{MHz}$ sensors: $\Delta m=-18.0 \cdot \Delta f$ based on $\Delta f_{\rho=0}$ averaged from $n=3,5$, and 7. $\Delta m^{\mathrm{SPR}}$ was determined as detailed in section S12 in the Supporting Information. All solutions contained $10 \mathrm{mM}$ Bis-Tris, $\mathrm{pH}(\mathrm{pD})=7.0,150 \mathrm{mM} \mathrm{NaCl}$, and $2.5 \mathrm{mM} \mathrm{CaCl}_{2}$.

inspecting $\Delta D_{\eta=0}$ at $n=3$, we observe that most of the energy dissipated is mechanical in nature, which is in agreement with the expected strong viscoelastic character of the adsorbate system. Note that $\Delta D_{\eta=0}$ not only reflects the mechanical properties of the linker, as in the cases of $\mathrm{SiO}_{2}$ and $\mathrm{Au}$ NPs, but also reflects the mechanical properties of the vesicles themselves. Altogether, these results show that the kinematicviscosity-matching approach seems capable to provide valuable information beyond rigid systems to systems with appreciable viscoelastic character.

The principles introduced above to determine the dry mass of adsorbed NPs are expected to also apply to adsorbed polymer films, ${ }^{73}$ given that they are in a regime with sufficiently small viscoelastic contributions, i.e., where the Sauerbrey equation (eq 1) is applicable not only for the dry mass but also the liquid coupled to the adsorbed polymer. This was tested by investigating electrostatically driven adsorption of HA to a PLL-coated sensor (Figure 7a), using the aforementioned strategy of kinematic-viscosity matching. Extrapolation of the frequency response in $\mathrm{D}_{2} \mathrm{O}$ and $\nu_{\text {matched }}$ buffers toward $\rho=0$ yields an intercept $\Delta f_{\rho=0}$ of $-29.8 \pm 2.2$ $\mathrm{Hz}$ with essentially no overtone dependence for $\Delta f_{\rho=0}$ values (Figure $7 \mathrm{~b}$ ). Additionally, conversion of $\Delta f_{\rho=0}$ into mass using eq 1 perfectly agrees with the mass obtained by employing complementary SPR measurements (see Figure $7 \mathrm{~d}$ and section S12 in the Supporting Information), confirming the validity of the extrapolation approach to determine the dry mass. Additionally, the mechanically dissipated energy represented by $\Delta D_{\eta=0}$ is less than half of the total dissipation, demonstrating that the majority of the dissipated energy is liquid-related in nature. For the applicability of the Sauerbrey equation on polymeric adlayers, it has been suggested that the dissipation-to-frequency ratio should be much smaller than 0.4 $\times 10^{-6} \mathrm{~Hz}^{-1}$. Although $\Delta D_{\mathrm{H}_{2} \mathrm{O}} / \Delta f_{\mathrm{H}_{2} \mathrm{O}}$ upon $\mathrm{HA}$ adsorption $\left(\approx 0.11 \times 10^{-6} \mathrm{~Hz}^{-1}\right)$ is indeed smaller than this threshold, it is on the same order and thus does not completely meet this criterion. Still, the perfect agreement of $\Delta f_{\rho=0}$ with the mass determined using SPR suggests that the kinematic-viscositymatching approach is applicable beyond perfectly rigid systems also for polymeric adlayers as for NPs.

\section{CONCLUSIONS}

Taken together, our results demonstrate that the proposed kinematic-viscosity-matching approach makes it possible to determine the (dry) molecular mass of both nanoscale 
adsorbates of arbitrary shape as well as polymeric adlayers, even for systems with appreciable viscoelastic contributions, and that the mechanically dissipated energy associated with the adsorbate and/or its attachment can be specifically quantified. This approach has been theoretically validated for firmly attached rigid adsorbates. While for biological soft matter the theoretical representation is far from trivial, our experiments indicate that for many such systems the approach proposed yields dry mass estimations within the uncertainty limits of the complementary methods used to verify the quantification of the adsorbed mass. More specifically, the experiments show that at lower overtones the liquid-related contribution to the QCM signal appears to be more related to the shape of soft matter (which is often rather complex) than to its deformation due to the oscillation of the crystal. The results also show that the dry-mass determination can be self-sufficiently validated based on the overtone dependence of the extrapolated $\Delta f_{\rho=0}$. For systems with weak to no measurable overtone dependence, the viscoelastic contribution is negligible, and thus the true dry mass can be determined based on any of the measured overtones. For systems with an appreciable overtone dependence, the mass determination at the lowest overtone number is the most reliable, highlighting one of the key discoveries in this work, namely, that the accuracy of the Sauerbrey equation is overtone dependent; i.e., the lower the overtone, the closer the Sauerbrey mass to the real mass. In addition, the approach does not require any a priori knowledge about the adsorbed material, which makes it unique in comparison with most commonly applied in situ methods, where ellipsometry and SPR stand out as the most reliable and commonly used. However, for accurate mass determination using these methods, at least the derivative of the refractive index with respect to the concentration of the adsorbate, $\mathrm{d} n / \mathrm{d} c$, must be known or independently determined. Additionally, in the case of SPR, also the film thickness and the decay length of the evanescent sensing field have to be determined independently. Finally, the possibility to simultaneously determine the dry mass and mechanical properties of nanosized adsorbates as well as the liquid contributions using a single method and on the same spatial scale provides a unique opportunity to potentially extract more detailed information about the adsorbates, such as investigating orientation, spatial distribution, and binding strength of adsorbates. Additionally, although demonstrated here using QCM, the kinematic-viscositymatching approach is potentially applicable beyond the QCM to a variety of mechanical resonators that operate in a liquid environment and could thus be utilized for single cell ${ }^{5}$ and even single virus ${ }^{74}$ mass determination.

\section{ASSOCIATED CONTENT}

\section{(s) Supporting Information}

The Supporting Information is available free of charge at https://pubs.acs.org/doi/10.1021/acs.jpcc.1c04502.

Detailed theoretical derivation; theoretical analysis on the applicablity of the kinematic-viscosity-matching approach on mechanical resonators other than the QCM; calculation of the physical properties of different $\mathrm{D}_{2} \mathrm{O} / \mathrm{H}_{2} \mathrm{O}$ and glycerol $/ \mathrm{H}_{2} \mathrm{O}$ mixtures; detailed methods and experimental procedure to measure the response in different liquids and for independent mass determination using measurements in air and SEM; experimental results for measurements with reference to air, polymer adsorption to APDMES-coated sensors, adsorption of carboxylated and PEGylated Au NPs, response at different overtones and $\mathrm{D}_{2} \mathrm{O}$ concentrations, lipid vesicles characterization and tethering, SPR calculations and results (PDF)

\section{AUTHOR INFORMATION}

\section{Corresponding Authors}

Antonius Armanious - Division of Nano and Biological Physics, Department of Physics, Chalmers University of Technology, 41296 Gothenburg, Sweden; Present Address: Laboratory of Food and Soft Materials, Department of Health Sciences and Technology, ETH Zürich, 8092 Zürich, Switzerland; ㅇo orcid.org/00000002-8809-4659; Email: armanioa@ethz.ch

Fredrik Höök - Division of Nano and Biological Physics, Department of Physics, Chalmers University of Technology, 41296 Gothenburg, Sweden; (1) orcid.org/0000-0003-19945015; Email: fredrik.hook@chalmers.se

\section{Authors}

Björn Agnarsson - Division of Nano and Biological Physics, Department of Physics, Chalmers University of Technology, 41296 Gothenburg, Sweden; 이이. orcid.org/0000-0003-33647196

Anders Lundgren - Department of Chemistry and Molecular Biology, University of Gothenburg, 40530 Gothenburg, Sweden; (1) orcid.org/0000-0002-8537-9974

Vladimir P. Zhdanov - Division of Nano and Biological Physics, Department of Physics, Chalmers University of Technology, 41296 Gothenburg, Sweden; Boreskov Institute of Catalysis, Russian Academy of Sciences, Novosibirsk 630090, Russia; 이이이.org/0000-0002-0167-8783

Complete contact information is available at: https://pubs.acs.org/10.1021/acs.jpcc.1c04502

\section{Author Contributions}

A.A. and F.H. conceived the idea. V.P.Z. developed the theoretical formalism. A.A., B.A., and A.L. designed and performed the experiments. A.A., V.P.Z., and F.H. wrote the paper with input from all authors.

\section{Notes}

The authors declare no competing financial interest.

\section{ACKNOWLEDGMENTS}

The authors thank Michael Rodal and the late Bengt Kasemo for valuable discussions and Andreas Dahlin and John Andersson for support with the SPR analysis. This work was performed in part at the Material Analysis Laboratory (CMAL) and infrastructure for nanofabrication (MC2) at Chalmers. The authors thank the Knut and Alice Wallenberg Foundation (2019-0577) and the Swedish Research Council (2018-04900, 2019-05071, and 2019-05215) for funding.

\section{REFERENCES}

(1) Sauerbrey, G. Verwendung von Schwingquarzen zur Wägung dünner Schichten und zur Mikrowägung. Eur. Phys. J. A 1959, 155, 206-222.

(2) Reviakine, I.; Johannsmann, D.; Richter, R. P. Hearing what you cannot see and visualizing what you hear: interpreting quartz crystal microbalance data from solvated interfaces. Anal. Chem. 2011, 83, $8838-8848$. 
(3) Ward, M. D.; Buttry, D. A. In-situ interfacial mass detection with piezoelectric transducers. Science 1990, 249, 1000-1007.

(4) Kosaka, P. M.; Pini, V.; Ruz, J. J.; Da Silva, R. A.; González, M. U.; Ramos, D.; Calleja, M.; Tamayo, J. Detection of cancer biomarkers in serum using a hybrid mechanical and optoplasmonic nanosensor. Nat. Nanotechnol. 2014, 9, 1047-1053.

(5) Martínez-Martín, D.; Fläschner, G.; Gaub, B.; Martin, S.; Newton, R.; Beerli, C.; Mercer, J.; Gerber, C.; Müller, D. J. Inertial picobalance reveals fast mass fluctuations in mammalian cells. Nature 2017, 550, 500-505.

(6) Tessier, L.; Patat, F.; Schmitt, N.; Feuillard, G.; Thompson, M. Effect of the generation of compressional waves on the response of the thickness-shear mode acoustic wave sensor in liquids. Anal. Chem. 1994, 66, 3569-3574.

(7) Rodahl, M.; Höök, F.; Krozer, A.; Brzezinski, P.; Kasemo, B. Quartz crystal microbalance setup for frequency and Q-factor measurements in gaseous and liquid environments. Rev. Sci. Instrum. 1995, 66, 3924-3930.

(8) Kanazawa, K. K.; Gordon, J. G. Frequency of quartz microbalance in contact with liquid. Anal. Chem. 1985, 57, 17701771

(9) Höök, F.; Kasemo, B.; Nylander, T.; Fant, C.; Sott, K.; Elwing, $\mathrm{H}$. Variations in coupled water, viscoelastic properties and film thickness of a Mefp-1 protein film during adsorption and crosslinking: a QCM-D, ellipsometry and SPR study. Anal. Chem. 2001, 73, 5796-5804.

(10) Wolff, O.; Seydel, E.; Johannsmann, D. Viscoelastic properties of thin films studied with quartz crystal resonators. Faraday Discuss. 1997, 107, 91-104.

(11) Voinova, M. V.; Rodahl, M.; Jonson, M.; Kasemo, B. Viscoelastic acoustic response of layered polymer films at fluid-solid interfaces: continuum mechanics approach. Phys. Scr. 1999, 59, 391396.

(12) Johannsmann, D. Viscoelastic, mechanical, and dielectric measurements on complex samples with the quartz crystal microbalance. Phys. Chem. Chem. Phys. 2008, 10, 4516-4534.

(13) Arazoe, H.; Miyajima, D.; Akaike, K.; Araoka, F.; Sato, E.; Hikima, T.; Kawamoto, M.; Aida, T. An autonomous actuator driven by fluctuations in ambient humidity. Nat. Mater. 2016, 15, 10841089.

(14) Liu, P.; Song, M.; Zhao, T.; Gunthe, S. S.; Ham, S.; He, Y.; Qin, Y. M.; Gong, Z.; Amorim, J. C.; Bertram, A. K.; Martin, S. T. Resolving the mechanisms of hygroscopic growth and cloud condensation nuclei activity for organic particulate matter. Nat. Commun. 2018, 9, 4076.

(15) Reinisch, L.; Kaiser, R. D.; Krim, J. Measurement of protein hydration shells using a quartz microbalance. Phys. Rev. Lett. 1989, 63, $1743-1746$

(16) Fu, Y.; Jiang, Y.-B.; Dunphy, D.; Xiong, H.; Coker, E.; Chou, S. S.; Zhang, H.; Vanegas, J. M.; Croissant, J. G.; Cecchi, J. L.; Rempe, S. B.; Brinker, C. J. Ultra-thin enzymatic liquid membrane for $\mathrm{CO}_{2}$ separation and capture. Nat. Commun. 2018, 9, 990.

(17) Feng, S.; Bein, T. Growth of oriented molecular sieve crystals on organophosphonate films. Nature 1994, 368, 834-836.

(18) Shpigel, N.; Sigalov, S.; Malchik, F.; Levi, M. D.; Girshevitz, O.; Khalfin, R. L.; Aurbach, D. Quantification of porosity in extensively nanoporous thin films in contact with gases and liquids. Nat. Commun. 2019, 10, 4394.

(19) Walcarius, A.; Sibottier, E.; Etienne, M.; Ghanbaja, J. Electrochemically assisted self-assembly of mesoporous silica thin films. Nat. Mater. 2007, 6, 602-608.

(20) Trachsel, L.; Romio, M.; Grob, B.; Zenobi-Wong, M.; Spencer, N. D.; Ramakrishna, S. N.; Benetti, E. M. Functional nanoassemblies of cyclic polymers show amplified responsiveness and enhanced protein-binding ability. ACS Nano 2020, 14, 10054-10067.

(21) Richter, R. P.; Hock, K. K.; Burkhartsmeyer, J.; Boehm, H.; Bingen, P.; Wang, G.; Steinmetz, N. F.; Evans, D. J.; Spatz, J. P. Membrane-grafted hyaluronan films: a well-defined model system of glycoconjugate cell coats. J. Am. Chem. Soc. 2007, 129, 5306-5307.
(22) Shen, H.-H.; Leyton, D. L.; Shiota, T.; Belousoff, M. J.; Noinaj, N.; Lu, J.; Holt, S. A.; Tan, K.; Selkrig, J.; Webb, C. T.; Buchanan, S. K.; Martin, L. L.; Lithgow, T. Reconstitution of a nanomachine driving the assembly of proteins into bacterial outer membranes. Nat. Commun. 2014, 5, 5078.

(23) Dubacheva, G. V.; Araya-Callis, C.; Geert Volbeda, A.; Fairhead, M.; Codée, J.; Howarth, M.; Richter, R. P. Controlling multivalent binding through surface chemistry: model study on streptavidin. J. Am. Chem. Soc. 2017, 139, 4157-4167.

(24) Gutiérrez Sánchez, C.; Su, Q.; Schönherr, H.; Grininger, M.; Nöll, G. Multi-ligand-binding flavoprotein dodecin as a key element for reversible surface modification in nano-biotechnology. ACS Nano 2015, 9, 3491-3500.

(25) Parveen, N.; Rydell, G. E.; Larson, G.; Hytönen, V. P.; Zhdanov, V. P.; Höök, F.; Block, S. Competition for membrane receptors: norovirus detachment via lectin attachment. J. Am. Chem. Soc. 2019, 141, 16303-16311.

(26) Di Iorio, D.; Verheijden, M. L.; Van Der Vries, E.; Jonkheijm, P.; Huskens, J. Weak multivalent binding of influenza hemagglutinin nanoparticles at a sialoglycan-functionalized supported lipid bilayer. ACS Nano 2019, 13, 3413-3423.

(27) Mateos-Gil, P.; Tsortos, A.; Velez, M.; Gizeli, E. Monitoring structural changes in intrinsically disordered proteins using QCM-D: Application to the bacterial cell division protein ZipA. Chem. Commun. 2016, 52, 6541-6544.

(28) Roach, P.; Farrar, D.; Perry, C. C. Interpretation of protein Adsorption: surface-induced conformational changes. J. Am. Chem. Soc. 2005, 127, 8168-8173.

(29) Tsortos, A.; Papadakis, G.; Gizeli, E. Shear acoustic wave biosensor for detecting DNA intrinsic viscosity and conformation: A study with QCM-D. Biosens. Bioelectron. 2008, 24, 836-841.

(30) Papadakis, G.; Tsortos, A.; Gizeli, E. Acoustic characterization of nanoswitch structures: Application to the DNA holliday junction. Nano Lett. 2010, 10, 5093-5097.

(31) Bisker, G.; Dong, J.; Park, H. D.; Iverson, N. M.; Ahn, J.; Nelson, J. T.; Landry, M. P.; Kruss, S.; Strano, M. S. Protein-targeted corona phase molecular recognition. Nat. Commun. 2016, 7, 1-14.

(32) Schmudde, M.; Grunewald, C.; Goroncy, C.; Noufele, C. N.; Stein, B.; Risse, T.; Graf, C. Controlling the interaction and non-closepacked arrangement of nanoparticles on large areas. ACS Nano 2016, 10, 3525-3535.

(33) Tellechea, E.; Johannsmann, D.; Steinmetz, N. F.; Richter, R. P.; Reviakine, I. Model-independent analysis of QCM data on colloidal particle adsorption. Langmuir 2009, 25, 5177-5184.

(34) Olsson, A. L.; Quevedo, I. R.; He, D.; Basnet, M.; Tufenkji, N. Using the quartz crystal microbalance with dissipation monitoring to evaluate the size of nanoparticles deposited on surfaces. ACS Nano 2013, 7, 7833-7843.

(35) Gillissen, J. J. J.; Tabaei, S. R.; Jackman, J. A.; Cho, N.-J. A model derived from hydrodynamic simulations for extracting the size of spherical particles from the quartz crystal microbalance. Analyst 2017, 142, 3370-3379.

(36) Reviakine, I.; Gallego, M.; Johannsmann, D.; Tellechea, E. Adsorbed liposome deformation studied with quartz crystal microbalance. J. Chem. Phys. 2012, 136, 084702.

(37) Gillissen, J. J. J.; Jackman, J. A.; Tabaei, S. R.; Yoon, B. K.; Cho, N.-J. Quartz crystal microbalance model for quantitatively probing the deformation of adsorbed particles at low surface coverage. Anal. Chem. 2017, 89, 11711-11718

(38) Zou, T.; Sipponen, M. H.; Henn, A.; Österberg, M. Solventresistant lignin-epoxy hybrid nanoparticles for covalent surface modification and high-strength particulate adhesives. ACS Nano 2021, 15, 4811-4823.

(39) Pilkington, E. H.; Gustafsson, O. J. R.; Xing, Y.; HernandezFernaud, J.; Zampronio, C.; Kakinen, A.; Faridi, A.; Ding, F.; Wilson, P.; Ke, P. C.; et al. Profiling the serum protein corona of fibrillar human islet amyloid polypeptide. ACS Nano 2018, 12, 6066-6078.

(40) Melby, E. S.; Lohse, S. E.; Park, J. E.; Vartanian, A. M.; Putans, R. A.; Abbott, H. B.; Hamers, R. J.; Murphy, C. J.; Pedersen, J. A. 
Cascading effects of nanoparticle coatings: surface functionalization dictates the assemblage of complexed proteins and subsequent interaction with model cell membranes. ACS Nano 2017, 11, 54895499.

(41) Lochbaum, C. A.; Chew, A. K.; Zhang, X.; Rotello, V.; Van Lehn, R. C.; Pedersen, J. A. Lipophilicity of cationic ligands promotes irreversible adsorption of nanoparticles to lipid bilayers. ACS Nano 2021, 15, 6562-6572.

(42) Janshoff, A.; Galla, H. J.; Steinem, C. Piezoelectric mass-sensing devices as biosensors - An alternative to optical biosensors? Angew. Chem., Int. Ed. 2000, 39, 4004-4032.

(43) Patolsky, F.; Lichtenstein, A.; Willner, I. Detection of singlebase DNA mutations by enzyme-amplified electronic transduction. Nat. Biotechnol. 2001, 19, 253-257.

(44) Cooper, M. A.; Dultsev, F. N.; Minson, T.; Ostanin, V. P.; Abell, C.; Klenerman, D. Direct and sensitive detection of a human virus by rupture event scanning. Nat. Biotechnol. 2001, 19, 833-837. (45) Webster, A.; Vollmer, F.; Sato, Y. Probing biomechanical properties with a centrifugal force quartz crystal microbalance. Nat. Commun. 2014, 5, 5284.

(46) Suthar, J.; Parsons, E. S.; Hoogenboom, B. W.; Williams, G. R.; Guldin, S. Acoustic immunosensing of exosomes using a quartz crystal microbalance with dissipation monitoring. Anal. Chem. 2020, 92, 4082-4093.

(47) Richter, R. P.; Mukhopadhyay, A.; Brisson, A. Pathways of lipid vesicle deposition on solid surfaces: a combined QCM-D and AFM study. Biophys. J. 2003, 85, 3035-3047.

(48) Reimhult, E.; Larsson, C.; Kasemo, B.; Höök, F. Simultaneous surface plasmon resonance and quartz crystal microbalance with dissipation monitoring measurements of biomolecular adsorption events involving structural transformations and variations in coupled water. Anal. Chem. 2004, 76, 7211-7220.

(49) van der Meulen, S. A. J.; Dubacheva, G. V.; Dogterom, M.; Richter, R. P.; Leunissen, M. E. Quartz crystal microbalance with dissipation monitoring and spectroscopic ellipsometry measurements of the phospholipid bilayer anchoring stability and kinetics of hydrophobically modified DNA oligonucleotides. Langmuir 2014, 30, 6525-6533.

(50) Landau, L. D.; Lifshitz, E. M. Fluid Mechanics; Pergamon Press, 1987; Chapter II, pp 44-94.

(51) Perrault, S. D.; Chan, W. C. Synthesis and surface modification of highly monodispersed, spherical gold nanoparticles of 50-200 nm. J. Am. Chem. Soc. 2009, 131, 17042-17043.

(52) Johannsmann, D.; Reviakine, I.; Richter, R. P. Dissipation in films of adsorbed nanospheres studied by quartz crystal microbalance (QCM). Anal. Chem. 2009, 81, 8167-8176.

(53) Adamczyk, Z.; Sadowska, M. Hydrodynamic Solvent Coupling Effects in Quartz Crystal Microbalance Measurements of Nanoparticle Deposition Kinetics. Anal. Chem. 2020, 92, 3896-3903.

(54) Adamczyk, Z.; Sadowska, M.; Żeliszewska, P. Applicability of QCM-D for Quantitative Measurements of Nano- And Microparticle Deposition Kinetics: Theoretical Modeling and Experiments. Anal. Chem. 2020, 92, 15087-15095.

(55) Vázquez-Quesada, A.; Schofield, M. M.; Tsortos, A.; MateosGil, P.; Milioni, D.; Gizeli, E.; Delgado-Buscalioni, R. Hydrodynamics of Quartz-Crystal-Microbalance DNA Sensors Based on Liposome Amplifiers. Phys. Rev. Appl. 2020, 13, 064059.

(56) Meléndez, M.; Vázquez-Quesada, A.; Delgado-Buscalioni, R. Load Impedance of Immersed Layers on the Quartz Crystal Microbalance: a Comparison with Colloidal Suspensions of Spheres. Langmuir 2020, 36, 9225-9234.

(57) Daikhin, L.; Gileadi, E.; Katz, G.; Tsionsky, V.; Urbakh, M.; Zagidulin, D. Influence of roughness on the admittance of the quartz crystal microbalance immersed in liquids. Anal. Chem. 2002, 74, 554561.

(58) Armanious, A.; Aeppli, M.; Sander, M. Dissolved organic matter adsorption to model surfaces: adlayer formation, properties, and dynamics at the nanoscale. Environ. Sci. Technol. 2014, 48, 9420-9.
(59) Duval, J. F.; Wilkinson, K. I.; Van Leeuwen, H. P.; Buffle, J. Humic substances are soft and permeable: Evidence from their electrophoretic mobilities. Environ. Sci. Technol. 2005, 39, 64356445.

(60) Sutton, R.; Sposito, G. Molecular structure in soil humic substances: The new view. Environ. Sci. Technol. 2005, 39, 90099015.

(61) Armanious, A.; Aeppli, M.; Jacak, R.; Refardt, D.; Sigstam, T.; Kohn, T.; Sander, M. Viruses at solid-water interfaces: a systematic assessment of interactions driving adsorption. Environ. Sci. Technol. 2016, 50, 732-743.

(62) Klüpfel, L.; Piepenbrock, A.; Kappler, A.; Sander, M. Humic substances as fully regenerable electron acceptors in recurrently anoxic environments. Nat. Geosci. 2014, 7, 195-200.

(63) Latch, D. E.; McNeill, K. Microheterogeneity of singlet oxygen distributions in irradiated humic acid solutions. Science 2006, 311, $1743-1747$.

(64) Ellis, J. S.; Thompson, M. Slip and coupling phenomena at the liquid-solid interface. Phys. Chem. Chem. Phys. 2004, 6, 4928-4938.

(65) Johannsmann, D.; Reviakine, I.; Rojas, E.; Gallego, M. Effect of sample heterogeneity on the interpretation of $\mathrm{QCM}(-\mathrm{D})$ data: comparison of combined quartz crystal microbalance/atomic force microscopy measurements with finite element method modeling. Anal. Chem. 2008, 80, 8891-8899.

(66) Friedt, J.-M.; Francis, L.; Choi, K.-H.; Frederix, F.; Campitelli, A. Combined atomic force microscope and acoustic wave devices: Application to electrodeposition. J. Vac. Sci. Technol. A 2003, 21, $1500-1505$.

(67) Friedt, J.-M.; Choi, K. H.; Frederix, F.; Campitelli, A. Simultaneous AFM and QCM measurements: methodology validation using electrodeposition. J. Electrochem. Soc. 2003, 150, H229.

(68) Benkoski, J. J.; Höök, F. Lateral mobility of tethered vesicleDNA assemblies. J. Phys. Chem. B 2005, 109, 9773-9779.

(69) Kučerka, N.; Tristram-Nagle, S.; Nagle, J. F. Structure of fully hydrated fluid phase lipid bilayers with monounsaturated chains. $J$. Membr. Biol. 2006, 208, 193-202.

(70) Rastogi, R. P.; Skukla, P. C.; Yadava, B. Membrane Permeability of Heavy Water. Biochim. Biophys. Acta, Biomembr. 1971, 249, 454461.

(71) Roberts, K.; Alberts, B.; Johnson, A.; Walter, P.; Hunt, T. Molecular Biology of the Cell; Garland Science: New York, 2014; Chapter 11, pp 597-640.

(72) Bränden, M.; Tabaei, S. R.; Fischer, G.; Neutze, R.; Höök, F. Refractive-Index-Based Screening of Membrane-Protein Mediated Transfer Across Biological Membranes. Biophys. J. 2010, 99, 124133.

(73) Daikhin, L.; Urbakh, M. Effect of surface film structure on the quartz crystal microbalance response in liquids. Langmuir 1996, 12, $6354-6360$

(74) Dolai, S.; Tabib-Azar, M. $433 \mathrm{MHz}$ Lithium Niobate microbalance aptamer-coated whole Zika virus sensor with $370 \mathrm{~Hz} /$ ng sensitivity. IEEE Sens. J. 2020, 20, 4269-4274. 